\title{
Real-time fMRI neurofeedback training to improve eating behavior by self-regulation of the dorsolateral prefrontal cortex: a randomized controlled trial in overweight and obese subjects
}

\author{
Simon H. Kohl ${ }^{1,2 *}$, Ralf Veit ${ }^{1,3,4 *}$, Maartje S. Spetter ${ }^{1,5}$, Astrid Günther ${ }^{1}$, Andriani \\ Rina $^{1,4,6}$, Michael Lührs ${ }^{7,8}$, Niels Birbaumer ${ }^{1,9}$, Hubert Preissl ${ }^{3,10 \#}$, Manfred Hallschmid ${ }^{1,3}$ \\ ${ }^{1}$ Institute of Medical Psychology and Behavioral Neurobiology, University of Tübingen, \\ Germany \\ ${ }^{2}$ Cognitive Neuroscience, Institute of Neuroscience and Medicine (INM-3), Jülich Research \\ Center, Jülich, Germany \\ ${ }^{3}$ Institute for Diabetes Research and Metabolic Diseases of the Helmholtz Center Munich at \\ the University of Tübingen; German Center for Diabetes Research (DZD), Tübingen, \\ Germany \\ ${ }^{4}$ High-Field Magnetic Resonance Center, Max Planck Institute for Biological Cybernetics, \\ Tübingen, Germany \\ ${ }^{5}$ School of Psychology, University of Birmingham, Edgbaston, Birmingham, UK \\ ${ }^{6}$ Brigham and Women's Hospital, Harvard Medical School, Boston, MA, USA \\ ${ }^{7}$ Brain Innovation B.V., Research department, Maastricht, Netherlands \\ ${ }^{8}$ Faculty of Psychology and Neuroscience, Department of Cognitive Neuroscience, \\ Maastricht University, Netherlands \\ ${ }^{9}$ Wyss Center for Bio and Neuroengineering, Geneva, 1202, Switzerland \\ ${ }^{10}$ Institute of Pharmaceutical Sciences, Interfaculty Centre for Pharmacogenomics and \\ Pharma Research, Department of Pharmacy and Biochemistry, University of Tübingen, \\ Tübingen, Germany
}

*These authors contributed equally to the work.

Number of tables: 1; number of figures: 9; one appendix with 5 supplementary tables Abstract word count: 335; highlights word count: 54; word count: 7871

Clinical Trials Registration: This trial has been registered at the US National Institutes of Health (ClinicalTrials.gov, ref. no: NCT02148770;

https://clinicaltrials.gov/ct2/show/NCT02148770)

\#Corresponding author: H.P., Institute for Diabetes Research and Metabolic Diseases of the Helmholtz Center Munich at the University of Tübingen, Otfried-Müller-Str. 47, 72076 Tübingen, Germany; Phone: +49-7071-2987704, Fax: +49-7071-295706; E-mail: hubert.preissl@uni-tuebingen. 


\section{Abstract}

Obesity is associated with altered responses to food stimuli in prefrontal brain networks that mediate inhibitory control of ingestive behavior. In particular, activity of the dorsolateral prefrontal cortex (dlPFC) is reduced in obese compared to normal-weight subjects and has been linked to the success of weight-loss dietary interventions. In a randomized controlled trial in overweight/obese subjects, we investigated the effect on eating behavior of volitional up-regulation of dlPFC activity via real-time functional magnetic resonance imaging (fMRI) neurofeedback training.

Thirty-eight overweight or obese subjects (BMI $25-40 \mathrm{~kg} / \mathrm{m}^{2}$ ) took part in fMRI neurofeedback training with the aim of increasing activity of the left dlPFC (dlPFC group; $n=17$ ) or of the visual cortex (VC/control group; $n=21$ ). Participants were blinded to group assignment. The training session took place on a single day and included three training runs of six trials of up-regulation and passive viewing. Food appraisal and snack intake were assessed at screening, after training, and in a follow-up session four weeks later.

Participants of both groups succeeded in up-regulating activity of the targeted brain area. However, participants of the control group also showed increased left dlPFC activity during up-regulation. Functional connectivity between dIPFC and ventromedial PFC, an area that processes food value, was generally increased during up-regulation compared to passive viewing. At follow-up compared to baseline, both groups rated pictures of high-, but not low-calorie foods as less palatable and chose them less frequently. Actual snack intake remained unchanged but palatability and choice ratings for chocolate cookies decreased after training.

We demonstrate that one session of fMRI neurofeedback training enables individuals with increased body weight to up-regulate activity of the left dlPFC. Behavioral effects were observed in both groups, which might have been due to dlPFC co-activation in the control group and, in addition, unspecific training effects. Improved dlPFC-vmPFC functional connectivity furthermore suggested enhanced food intake-related control mechanisms. Neurofeedback training might support therapeutic strategies aiming at improved self-control in obesity, although the respective contribution of area-specific mechanisms and general regulation effects is in need of further investigation.

Keywords: Real-time functional magnetic resonance imaging, neurofeedback, overweight, obesity, dorsolateral prefrontal cortex, eating behavior. 


\section{Introduction}

Obesity is currently one of the major global health concerns. According to estimates based on data from 195 countries (Ashfin et al., 2017), 108 million children and 604 million adults were obese in 2015, and the prevalence of obesity has doubled in more than 70 countries and increased in most other countries since 1980. In Germany, almost 25\% of the population are obese, and in 2008, direct and indirect costs attributed to excess body weight amounted to $€$ 16.8 billion (Lehnert et al., 2013). Obesity and associated diseases like type 2 diabetes, metabolic syndrome, cardiovascular diseases and certain cancers (Bray, 2004) significantly increase all-cause mortality (Flegal et al., 2013). The long-term inefficacy of behavioral and pharmacological interventions to achieve significant but also sustained weight loss (Jensen et al., 2014) highlights the need for innovative approaches to improve control of body weight.

Obesity is associated with increased responsiveness to food stimuli (Nijs et al., 2010), attenuated inhibitory control (Bartholdy et al., 2016; Hege et al., 2015; Lavagnino et al., 2016) and increased intake of high-calorie and energy-dense palatable food (Berthoud and Zheng, 2012). On the neural level, obese individuals display hyperactivity in response to food stimuli in brain areas associated with reward, emotion, interoception and gustatory processing (e.g., striatum, orbitofrontal cortex, insula and amygdala), whereas the activity of areas that mediate cognitive control, particularly inhibitory control, is attenuated (e.g. Batterink et al., 2010; Le et al., 2007, 2006; Volkow et al., 2009; for review see Carnell et al., 2012). Thus, obesity is associated with reduced activity of the left dorsolateral prefrontal cortex (dlPFC) and the left insula in response to food images (Brooks et al., 2013), suggesting that improving the functionality of prefrontal cognitive control regions in obese individuals may help prevent overeating. Fittingly, self-control of food choice depends on a prefrontal network with the dIPFC as a core hub (Hollmann et al., 2012; Yokum and Stice, 2013), and successful dieting is associated with increased activation of the superior frontal gyrus as well as the dlPFC in response to food images and to food intake (DelParigi et al., 2007; Jensen \& Kirwan, 2015; Le et al., 2007). Frontocortical activation has also been reported to be stronger in obese subjects capable of maintaining decreased body weight (McCaffery et al., 2009). Hare and coworkers (2009) found that the dlPFC exerts top-down control over the ventromedial prefrontal PFC (vmPFC), which processes the subjective value of food items. In accordance, activity of the dIPFC, but also vmPFC and dorsomedial PFC as well as dlPFC-vmPFC connectivity predicts the success of dietary weight-loss interventions (Weygandt et al., 2013, 2015). 
Neurofeedback based on real-time functional magnetic resonance imaging (rt-fMRI) provides online feedback of brain activity and enables volitional regulation of the activity of circumscribed brain areas (Weiskopf, 2012; Weiskopf et al., 2007). Furthermore, rt-fMRI neurofeedback can induce behavioral effects in healthy but also clinical populations (for recent reviews see Sitaram et al., 2017; Thibault et al., 2017). We have recently demonstrated in pilot experiments that obese individuals are able to self-regulate disorder-specific brain areas and networks (Frank et al., 2012; Spetter et al., 2017). Therefore, in the present clinical trial we implemented a neurofeedback protocol to up-regulate dlPFC activity and, consequently, improve self-regulation of eating in the presence of food cues.

Of particular relevance for our approach was the inclusion of a suitable control condition. To date, most clinical rt-fMRI neurofeedback studies have either relinquished a control group or applied mental imagery or sham feedback, i.e., simulated feedback or prerecorded feedback of another participant (see Thibault et al., 2017). Due to absent contingency between the participant's mental efforts and the provided feedback, such approaches can strongly reduce motivation (Johnson et al., 2012). In line with recent clinical neurofeedback trials (e.g., Mehler et al., 2018; Young et al., 2017), we decided to provide feedback from regions that are not primarily related to control of eating behavior. While our intervention group received feedback on dlPFC activity (dlPFC group), the control group received feedback from primary and secondary visual cortex (VC/control group), an area that has already been successfully used as a target of rt-fMRI neurofeedback training (Scharnowski et al., 2012; Shibata et al., 2011). Behavioral effects of the training were assessed by questionnaire and real-life food choice tasks. Based on our previous studies (Spetter et al., 2017) we hypothesized that participants are able to up-regulate dlPFC activity after one session of neurofeedback training, with associated changes in food choices and preferences. In the participants of the control group, we expected up-regulation of visual areas, but no effect on food choices or food evaluation. We also expected increased activity in insula and striatum across groups because these areas respond to neurofeedback training independent of the target region (Emmert et al., 2016).

\section{Methods}

\subsection{Participants}

Participants were recruited from the community via announcements and e-mail lists. Eligibility was ensured by a telephone screening and a subsequent session (see below). Inclusion criteria were body mass index (BMI) between 25 and $40 \mathrm{~kg} / \mathrm{m}^{2}$, elevated body fat, 
no weight loss of over $5 \mathrm{~kg}$ within three months before screening, no eating disorders, psychiatric or neurological diseases, no drug or alcohol abuse, no smoking and no contraindications for MRI, e.g., metallic parts in the body or claustrophobia. In total, forty overweight or obese but otherwise healthy subjects were enrolled in the study, yielding 35 complete data sets after dropouts (see Figure 1 for CONSORT flow diagram). Each participant gave written informed consent and received a financial compensation of $100 €$ for attending all sessions or $10 € / \mathrm{h}$ in case of discontinuation. The study protocol was approved by the local ethics committee and registered under NCT02148770 on clinicaltrials.gov.

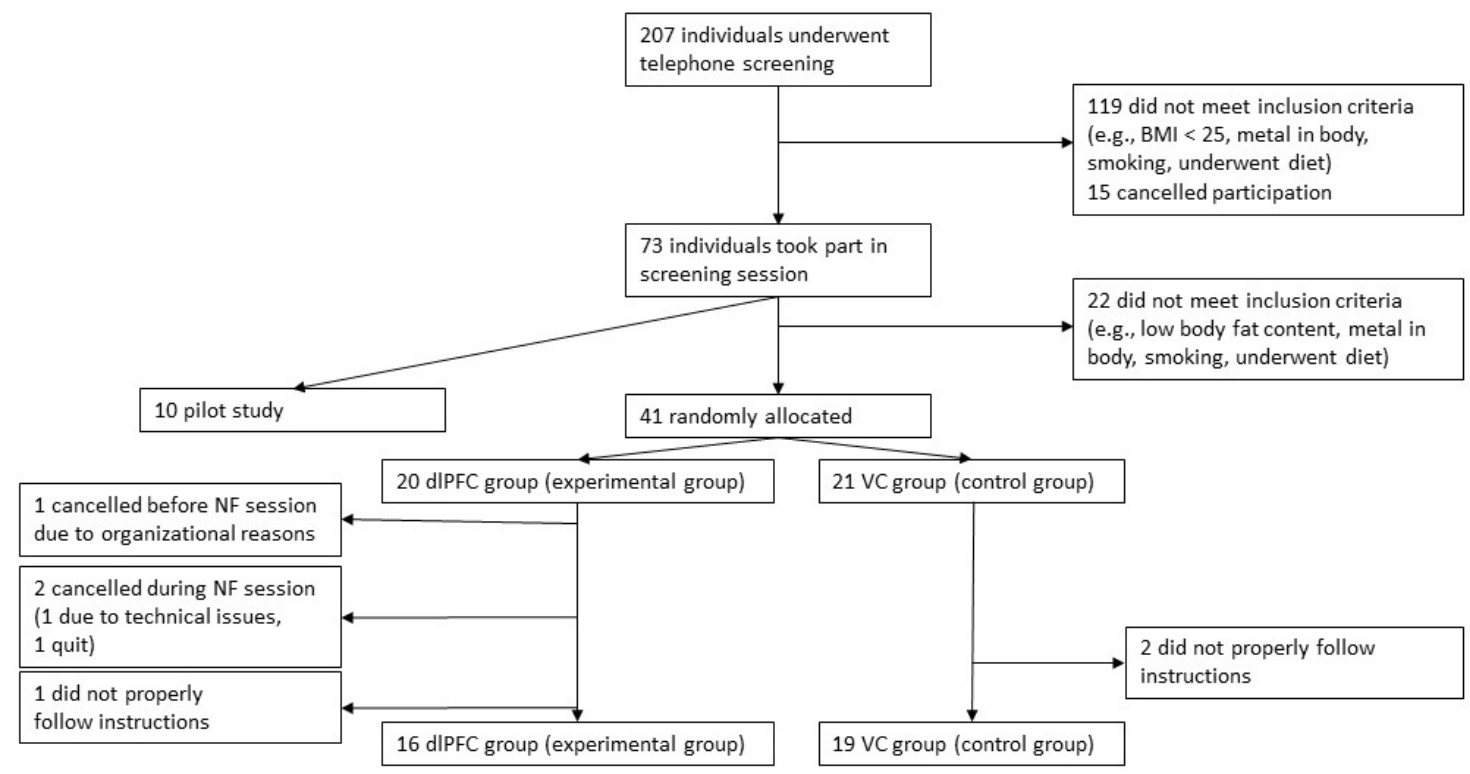

Figure 1. CONSORT flow diagram.

After inclusion, participants were randomly allocated to the dlPFC or VC group using a minimization program (South East Wales Trials Unit; SEWTU) that balanced the groups for sex and responses in the Eating Disorder Examination questionnaire (EDE-Q; Hilbert \& Tuschen-Caffier, 2011). Baseline characteristics of both groups are shown in Table 1 of the Results section.

\subsection{Study design}

We applied a randomized controlled between-subjects design including three appointments, i.e., a two-hour screening session, the rt-fMRI neurofeedback session and a follow-up session four weeks after the neurofeedback session (Figure 2). All three sessions took part in the morning. Participants were instructed to attend the lab in the fasted state, and upon 
arrival of the subject, we ensured that she/he had abstained from food intake for at least two hours. Participants moreover completed visual analogue scales assessing hunger and satiety. Subjects were blinded with regard to which group they belonged to and were unblinded only after they had completed the study. Due to the experimental setup it was not possible to also blind the experimenters during the neurofeedback session. However, behavioral assessments at screening and follow-up were conducted by an experimenter blinded to group assignments.

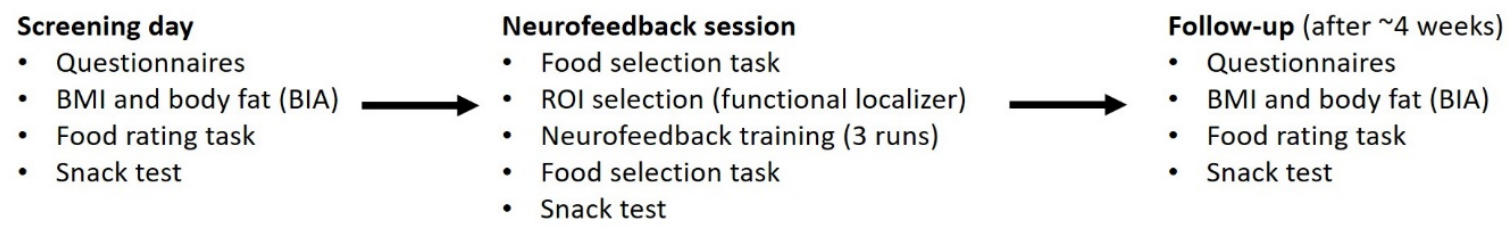

Figure 2. Study design. Participants underwent three experimental sessions, i.e., screening, neurofeedback session and a follow-up session four weeks after the neurofeedback session.

\subsubsection{Screening session}

In the screening session, a blood sample was drawn to determine fasting blood glucose, insulin and cholesterol. Hb1Ac values were obtained to exclude subjects with impaired glucose homeostasis or diabetes. Body height and weight were assessed and body composition was determined via bioelectrical impedance analysis (BIA; BIA 2000-M, Data Input GmbH, Pöcking, Germany) and subjects were included if their body fat content was above the 90th percentile. A screening questionnaire to assess demographic variables and check for potential exclusion criteria, the EDE-Q (Hilbert \& Tuschen-Caffier, 2011), the Power of Food Scale (PFS; Lowe \& Butryn, 2007) and the German version of the Three Factor Eating Questionnaire (TFEQ) - Fragebogen zum Essverhalten (FEV; Pudel \& Westenhoefer, 1989) were filled in.

\section{Food rating task}

A computer task divided in three parts was performed (food rating task). Participants first rated the subjective tastiness and healthiness of 50 high-calorie (e.g., fries or chocolate bars) and 45 low-calorie food items (e.g., fruits and vegetables) in two separate blocks on a 5point scale $(1=$ not at all tasty/very unhealthy, $2=$ not tasty/unhealthy, $3=$ neutral, $4=$ tasty/healthy, 5 very tasty/healthy). Based on the healthiness and tastiness rating a personalized neutral reference item was selected (item rated neutral in both taste and health). In a following choice task, first this reference item was shown and then participants had to 
indicate for all 95 food pictures how much they preferred to eat this item compared to the neutral reference food on a 5 -point choice scale $(1=$ not at all, $2=$ a little, $3=$ neutral, $4=$ much, $5=$ very much). For stimulus presentation, the software Presentation (Neurobehavioral Systems Inc, www.neurobs.com) was used and food images were taken from a standardized picture set (http://nutritionalneuroscience.eu; Charbonnier et al., 2015). The task was based on the behavioral task designed by Hare et al. (2009) to evaluate selfcontrol in the context of food choices.

\section{Snack test}

For the covert investigation of snack intake, three plates were placed on a table that contained snacks different in taste but roughly comparable in calorie content and macronutrient composition. They were labeled snack A, B, and C, respectively. The three types were, “TUC Cracker Classic” (salty/savoury taste; Griesson-de Beukelaer, Polch, Germany, 488 kcal/100 g), "Rice Waffles" (bland taste; Continental Bakeries B.V., Dordrecht, The Netherlands, 389 kcal/100 g), and "Double Chocolate Cookies" (sweet taste; EDEKA, Hamburg, Germany, 503 kcal/100 g), all broken down into bite-sized pieces. Of each variety a considerable amount could be eaten without the plates appearing empty, to ensure that participants would not restrict snack intake based on whether the experimenter could see how much had been consumed. In addition, a glass of water was provided. The participant was instructed to taste and rate each type of cookie on a visual analogue scale assessing palatability, sweetness, saltiness and sourness, anchored at 0 (not at all) and $10 \mathrm{~cm}$ (very palatable/sweet/salty). The importance of giving accurate ratings was emphasized and participants were informed that during and after completion of the rating task they could eat as many snacks as they liked because any remaining snacks would be discarded, and were left alone for $10 \mathrm{~min}$. Snack intake was covertly measured by weighing before and after the test without awareness of the participant. This test to measure casual food intake is based on work by Higgs et al., (2008) and has repeatedly been successfully applied to assess changes in food intake (e.g., Hallschmid et al., 2012; Ott et al., 2013)

\subsubsection{Neurofeedback session}

At the start of the neurofeedback session, participants filled in an MRI safety questionnaire, the German short version of the Profile of Mood States (POMS, McNair, Lorr, \& Droppleman, 1981) and a questionnaire assessing expectations and motivation towards the neurofeedback training. The MRI session was divided into two parts. First, a functional scan 
was conducted, during which participants performed a simple binary food selection task, followed by an anatomical MRI measurement. Afterwards the participants left the scanner for the instructions on the neurofeedback training. Then the second part of the MRI session began, consisting of a functional localizer, the neurofeedback training and again the food selection task. Finally, the participant left the scanner for good and the session ended with two more questionnaires (debriefing questionnaire and POMS) and the snack test.

\section{Food selection task (pre and post neurofeedback)}

This task was used to assess behavioral effects of the intervention. It consisted of 48 trials, in which a high-calorie and a low-calorie food picture were presented next to each other on a screen for 5 sec. Participants had to decide via button press which food they preferred to eat at this moment without considering calories or health aspects. The food pictures (24 highand 24 low-calorie food items) displayed were selected individually based on the highest ratings in tastiness given by the participant during the screening session in the food rating task. Stimuli were presented on a screen using the program Psychtoolbox on Matlab (The Mathworks Inc, Natick, MA).

\section{Functional localizer}

The functional localizer was performed to individually determine the region of interest (ROI) for the neurofeedback training, i.e., left dIPFC or VC. In the functional localizer session, the participant saw pictures of personalised tempting palatable foods (based on taste ratings of the food rating task) and was instructed to down-regulate the urge for food. Hollmann et al., (2012) have reported stable dlPFC activation using this approach. For the functional localizer, the same procedure as for the neurofeedback training (see below) was used but with less trials and without providing feedback. It consisted of three trials of regulation and passive viewing (each 30s) interspered with rest blocks of $12 \mathrm{sec}$. After running the localizer, the computed statistical map was used to select the dIPFC (contrast up-regulation minus viewing) or VC (contrast up-regulation minus rest). A rectangular box extending over 3 slices was drawn in the left dlPFC or bilateral VC, respectively. Only voxels (clusters) exceeding a statistical threshold of $\mathrm{t}=3.1$ within the box were used for the training ROIs (see Figure 3 for the individual training ROIs). Another box of voxels with individually comparable size in white matter parietal regions was selected as a second ROI (ROI 2). The signal of ROI 2 was later used to control for global fluctuations of the blood oxygen leveldependent (BOLD) signal and other unspecific effects from the feedback signal. 


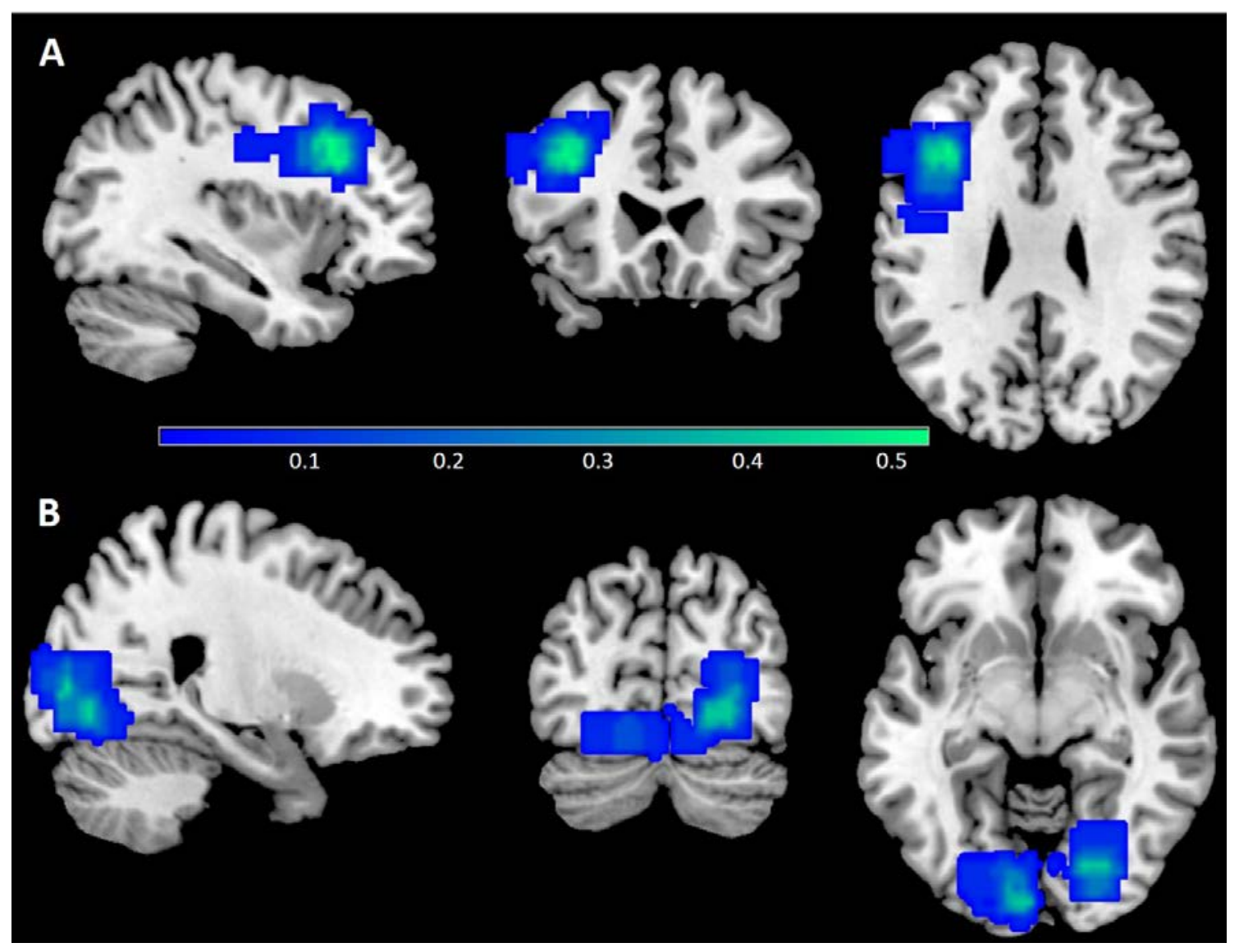

Figure 3. Average rt-fMRI neurofeedback ROIs. Average ROI selection in (A) the dIPFC group and (B) the VC group based on the functional localizer. The corresponding ROI masks were normalized into MNI space. Overlapping voxels are color coded activation map (blue-light green).

\section{Neurofeedback training}

Participants received standardized information and instruction based on Greer et al. (2014). They were told that the goal of the training was to increase activation of a specific brain area while they viewed pictures of tempting palatable foods. No specific self-regulation strategies were recommended (although participants might have been primed by the instructions given at the functional localizer session). Participants were rather encouraged to try out their own mental strategies and learn by trial and error how to increase the feedback signal, which represented the BOLD signal in the training ROI. Respective questionnaire responses indicated that participants indeed used different cognitive strategies to up-regulate brain activity (although often relying on mentally depreciating the viewed food items; Table S1), which is in line with previous observations that providing subjects with explicit cognitive strategies is not mandatory for successful regulation (Birbaumer et al., 2013; Shibata et al., 2011). Participants were also instructed to avoid motor or respiratory strategies and to 
consider that the feedback signal was approximately 4 to 6 sec delayed (i.e., by the physiological delay of the BOLD response).

\section{- Insert Supplementary Table S1 here -}

After subjects had re-entered the scanner and completed the functional localizer, neurofeedback training started. There were three training runs of 9 minutes with a short break after each run. Each run comprised six trials of $30 \mathrm{sec}$ passive viewing and $30 \mathrm{sec}$ upregulation of the training ROI, with a 12 sec rest period in-between and after each trial. During up-regulation and passive viewing, individually chosen high-calorie food pictures were presented that had received the highest palatability ratings during the screening session. Two black thermometer symbols on the left and respectively right side of the food picture provided feedback on the BOLD signal and a symbol next to the thermometer images indicated the type of the task (a plus sign during passive viewing and an upward arrow during up-regulation). Whenever the BOLD response of the training ROI increased by 0.05 percent compared to the passive-viewing condition, the thermometer bars rose by one of its ten levels. In order to adhere to operant learning principles (Sherlin et al., 2011; Strehl, 2014), this feedback threshold was individually adapted after each run depending on individual performance. If performance was so strong that the thermometer bar reached its limit, the threshold was increased stepwise to 0.075 or 0.1 percentage signal change per bin. This procedure implicated a maximal percent signal change (PSC) ranging between 0.5 and 1.0 percent; more details on the PSC calcuation are given in section 2.4.2. During passive viewing, the same pictures as during up-regulation were presented, but no feedback signal was presented (i.e., the thermometer bars were empty and did not change) and participants were told to view the food picture without trying to regulate. Stimuli were presented on a screen via a computer interface using the program Psychtoolbox on Matlab (see Figure 4). After neurofeedback training, participants again performed the food selection task while their brain responses were recorded to measure immediate effects of the training. 


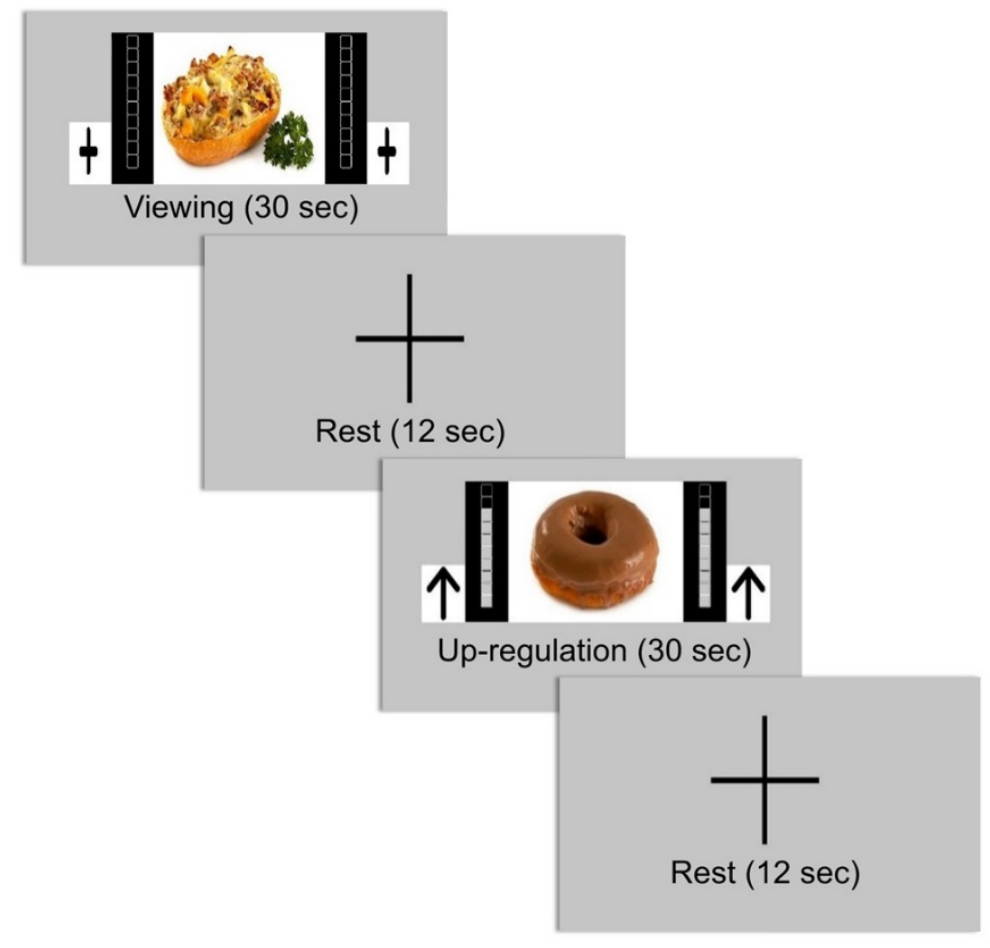

Figure 4. rt-fMRI neurofeedback training. BOLD signals were acquired via fMRI scans, processed in realtime using Turbo-BrainVoyager (v3.2; Brain Innovation B.V., Maastricht, The Netherlands) and presented as visual feedback on a stimulation computer. Visual feedback was provided only during up-regulation blocks in the form of thermometer bars indicating increases of the BOLD signal in the dorsolateral prefrontal cortex or visual cortex. During up-regulation blocks, participants were instructed to increase the thermometer bars, whereas during viewing blocks, they were instructed to passively look at the pictures without trying to increase the thermometer bars. Depicted in the figure are examples of visual stimuli shown during passive viewing, rest and up-regulation.

\subsubsection{Follow-up session}

The follow-up session was scheduled four weeks after the neurofeedback training (with some participants returning after five or six weeks due to individual time constraints). In general, the procedure of the follow-up session resembled that of the screening session. To measure long-term effects of neurofeedback, body composition and BMI were assessed again. Also, participants performed the food rating task and the snack test and completed the EDE as well as a questionnaire evaluating the neurofeedback training.

\subsection{MRI acquisition}

Functional and structural MRI images were obtained on a 3-Tesla MRI scanner (Siemens Magnetom Prisma, Siemens Healthcare, Erlangen, Germany) equipped with a 20-channel head coil (Siemens Magnetom Tim TRIO, Siemens Healthcare, Erlangen, Germany). For the T1-weighted anatomical scan the following parameters were used: repetition time (TR) $=2300 \mathrm{~ms}$, echo time $(\mathrm{TE})=4.18 \mathrm{~ms}$, flip angle $=9^{\circ}$, field of view $(\mathrm{FOV})=256 \times 256 \mathrm{~mm}$, 
176 axial slices and voxel size $=1 \times 1 \times 1 \mathrm{~mm}^{3}$. Functional images of the functional localizer and the neurofeedback runs were acquired with an EPI sequence using the following parameters: $\mathrm{TR}=1500 \mathrm{~ms}, \mathrm{TE}=30 \mathrm{~ms}$, adjusted flip angle $=79^{\circ}$, matrix size $=64 \times 64$, and 20 slices with a thickness of $4 \mathrm{~mm}$ and a gap of 13\%, resulting in a voxel size of $3.5 \times$ $3.5 \times 4.52 \mathrm{~mm}^{3}$. For the functional images obtained during the food selection task we used a whole brain coverage (40 slices) with the parameters: $\mathrm{TR}=2500 \mathrm{~ms}$, $\mathrm{TE}=30 \mathrm{~ms}$, flip angle $=70^{\circ}$, matrix size $=64 \times 64$ and 40 slices with a sickness of $3 \mathrm{~mm}$ and a gap of $20 \%$, resulting in a voxel size of $3 \times 3 \times 3.6 \mathrm{~mm}^{3}$.

\subsection{Data processing and analysis}

We applied a pre-specified analysis plan as stipulated within the framework of the BRAINTRAIN consortium (https://clinicaltrials.gov/ct2/show/NCT02148770). For online rt-fMRI data processing the software Turbo-BrainVoyager (v3.2; Brain Innovation B.V., Maastricht, The Netherlands) was used. Offline preprocessing and analyses of the imaging data were performed with SPM 12 (Wellcome Trust Centre for Neuroimaging, London, UK) and the CONN toolbox (version 17.f., Whitfield-Gabrieli \& Nieto-Castanon, 2012; http://www.nitrc.org/projects/conn) implemented in Matlab 2016b (The Mathworks Inc., Natick, MA, USA). Questionnaires and behavioral data were analyzed using IBM SPSS Statistics 22 (Armonk, NY, USA). All behavioral tests and questionnaires were checked for normality using Shapiro-Wilk tests and histograms. In case of violation of the normality assumption, non-parametric tests were applied. Outliers were removed from analyses if they exceeded 3 SDs from the mean. Data are presented as means \pm standard errors of the mean (SEM) except indicated otherwise. For all behavioral analyses a p-value below 0.05 was considered significant and in case of multiple testing Bonferroni correction was applied.

\subsubsection{Analysis of the food rating task}

The food rating task comprised three ratings (healthiness, tastiness and choice ratings) given at two time points (screening and follow-up). To analyze the effects of neurofeedback on food evaluation, mean healthiness and tastiness scores for high- and low-calorie food items, respectively, were compared between screening and follow-up. To analyze the effects on food choices, the percentage of times the participant chose the food item over the neutral reference food item (yes-decision; choice rating > 3) was calculated for high- and low-calorie food items and compared between screening and follow-up. To further analyze food choices, the percentage of yes-decisions was separately calculated for preferred (tastiness ratings > 3) high-calorie and non-preferred (tastiness ratings < 3) low-calorie food items, and 
compared between screening and follow-up. In order to render results at screening and follow-up comparable, percentages of yes-decisions at follow-up were calculated also relative to the total number of choices in the corresponding category during screening. Mixed ANOVAs with the between-subject factor group and the within-subject factors time and calorie content were performed to determine if the evaluation and choices of high- and lowcalorie food items changed from screening to follow-up.

\subsubsection{Real-time fMRI data processing (online analysis)}

During neurofeedback training the functional images were exported from the MRI console computer to another computer using a shared folder and analyzed in real time using the software TBV. On-line realignment, spatial smoothing $(9 \mathrm{~mm})$ and drift removal were performed and to avoid T1 saturation effects, the first ten images were excluded from the analysis. Using a General Linear Model (GLM) statistical maps were incrementally computed and updated. During neurofeedback training the mean BOLD signal from the training ROI and ROI 2 (control region) was extracted and the feedback signal (FB) was computed as the difference between the two ROI time-courses, normalized to the passive viewing condition using the formula:

$$
F B=\left(\frac{\text { training ROI }(\text { BOLDupreg }- \text { BOLDview })}{\text { training ROI(BOLDview })}-\frac{\text { ROI 2(BOLDupreg }- \text { BOLDview })}{\text { ROI 2(BOLDview })}\right) \times 100
$$

BOLDupreg represents the moving average over the last 4 TRs (6 sec) of the BOLD signal during the up-regulation condition, and BOLDview the mean BOLD signal of the previous phase of the passive viewing condition with a baseline shift of 4 TRs, thus reflecting the averaged time series of the last 16 scans of the passive viewing condition. To convert the FB value to a scale from 0 to 10 , a maximum PSC between 0.5 and 1 percent was used (see section 2.2.2). The feedback signal was converted into a thermometer image and sent to the stimulation computer. The image was continuously updated during presentation after each TR (every 1.5 sec; see Figure 4).

\subsubsection{Self-regulation performance}

To analyze if participants in both groups successfully up-regulated activity in the training ROI, BOLD time series were extracted from the individual training ROI and ROI 2 of each participant. For the localizer and each neurofeedback run the BOLD signal during upregulation and passive viewing was averaged over trials (discarding the first 4 scans of each 
trial to avoid effects of visually induced activity), and for both ROIs separate mean PSCs during up-regulation normalized to the passive viewing condition were calculated. The mean PSC of the ROI 2 was then subtracted from the PSC of the training ROI, resulting in a differential mean PSC (PSCdiff) for each participant in each neurofeedback run.

\subsubsection{Analysis of brain activity during neurofeedback and food selection task (offline analysis)}

Standard preprocessing steps were performed (realignment, coregistration, normalization, 9mm smoothing and high-pass filtering). Then two separate GLMs for neurofeedback regulation as well as for the food selection task were calculated for each participant, including regressors for the task as well as the realignment parameters as regressors of no interest. The model for neurofeedback regulation included the factors neurofeedback run and up-regulation vs. passive viewing and the model for the food selection task the factors choice (high- vs. low-calorie) and time. Afterwards whole brain second-level full factorial models with the within-subject factors neurofeedback run/time and the between-subject factor group including BMI and age as covariates were calculated. Results were considered significant at $\mathrm{p}<0.05$ FWE-corrected on cluster level. The primary voxel level threshold was set at $\mathrm{p}<0.001$ uncorrected. Due to head movements exceeding $3 \mathrm{~mm}$, two further participants, one from each group, were excluded from the offline fMRI analyses.

\subsubsection{Analysis of functional connectivity (offline analysis)}

Analyses of functional connectivity relied on bivariate correlations with hrf-weighting to define specific weights within conditions. We first denoised the functional images using the default settings of the CONN toolbox, including time courses of white matter and CSF BOLD signals, six realignment parameters and linear detrending. Main task effects were regressed out and a high-pass filter $(0.008 \mathrm{~Hz})$ was applied to remove physiological artifacts.

Functional connectivity ROIs comprised parts of the food appetitive network, areas that mediate self-control of eating behavior as well as brain areas involved in neurofeedback processing and self-regulation (anterior cingulate cortex, dorsal striatum, ventral striatum, dIPFC (BA46), insula, thalamus, inferior and superior parietal cortex, lateral occipital cortex, and vmPFC; Emmert et al., 2016; Sitaram et al., 2017). Most of the ROIs were anatomically selected using the Automated Anatomically Labeling atlas (AAL2; Tzourio-Mazoyer et al., 2002). The vmPFC was defined according to Hare et al., (2009). Only dlPFC and VC (i.e., the training ROIs) were defined using the pre-training functional localizer (see 2.2.2 
Neurofeedback session/Functional localizer). Since location and extension (number of voxels) of the dIPFC and visual areas slightly differed between subjects, we defined common dIPFC and visual area ROIs for the experimental and, respectively, control group (see Figure 3).

In order to investigate regulation-specific connectivity patterns, we compared the upregulation condition with the passive viewing condition independent of groups using firstlevel connectivity measures, with age and BMI as covariates. Moreover, we compared the dIPFC and the control group using a $2 \times 2$ mixed ANOVA with the between-subjects factor group and the within-subjects factor condition. In all second-level analyses, only FDRcorrected $p$-values were considered significant. 


\section{Results}

\subsection{Feasibility and self-regulation performance}

More than two thirds of the participants who attended the screening session were eligible for the study; only a few participants quit the study after screening or during neurofeedback, and all participants who finished neurofeedback also took part in the follow-up session (Figure 1), indicating high feasibility of our approach (see also 3.7).

Participants of both groups successfully up-regulated activity in the individual training ROIs (Figure 5). There was a significant time effect $(F(1,33)=4.407, p<0.01)$, indicating an increase in PSCdiff from the localizer session to the neurofeedback runs. There was no significant group effect and no respective interaction $(p>0.05)$. Contrast analyses revealed a significant difference between the localizer and the three neurofeedback runs ( $p$ $<0.05$ ), but no significant difference between the neurofeedback runs, neither across nor for the individual groups.

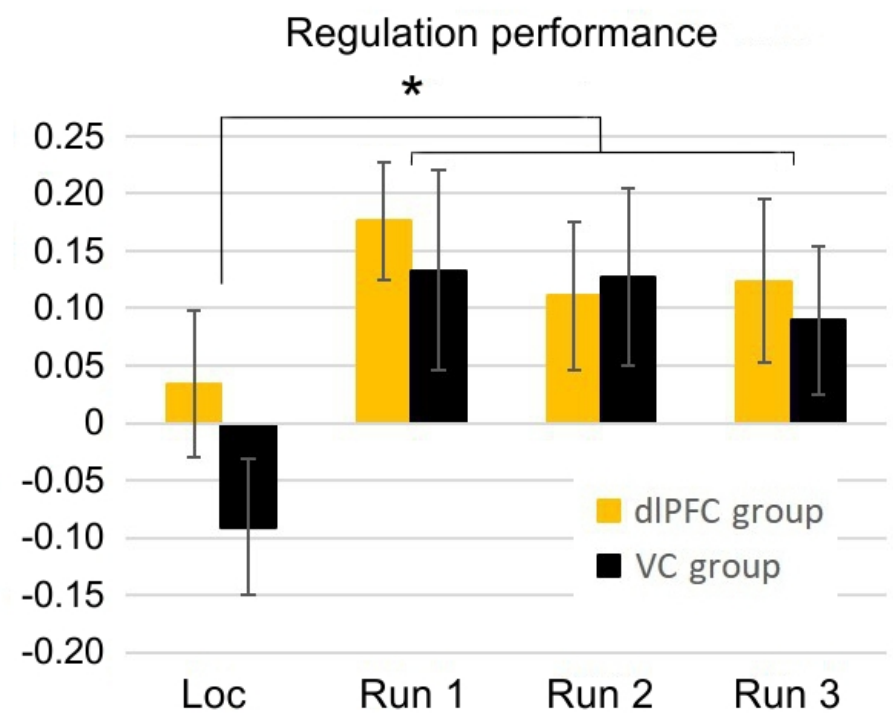

Figure 5. Regulation performance. Bars depict mean BOLD percent signal change $( \pm S E M)$ in the individual training ROIs of the two groups for the localizer session and the neurofeedback runs; ${ }^{*} p<0.05$.

Debriefing questionnaires indicated that neither directly after neurofeedback (52.94\%) nor in the follow-up session (55.88\%) participants were able to detect above chance levels if they were in the dIPFC or the VC group. 


\subsection{Brain activation during neurofeedback (offline analysis)}

The main effect of regulation (up-regulation - viewing) revealed a large significant cluster in the bilateral anterior insula extending to bilateral inferior frontal gyrus, thalamus and dorsal striatum and the right dlPFC. In addition, we found significant clusters in temporooccipital areas comprising the right superior temporal gyrus and bilateral middle temporal gyrus, extending to the middle occipital gyrus, i.e., the training ROI of the VC group. Moreover, a significant effect was observed in the left dlPFC, i.e., the training ROI of the dlPFC group. When comparing passive viewing with up-regulation, activation in the vmPFC as well as in the bilateral precuneus and angular gyrus (all $p<0.05$, FWE- corrected, $\mathrm{k}>10$ voxel) was observed. However, there were no significant group, time or interaction effects. Explorative group-specific analyses indicated that only the VC group, but not the dIPFC group showed a significant effect in the middle occipital gyrus, i.e., the training ROI of the VC group. Figure 6 and Tables S2-4 depict the main effect of up-regulation and simple main effects for both groups collapsed across all neurofeedback runs in a random-effects wholebrain analysis. 


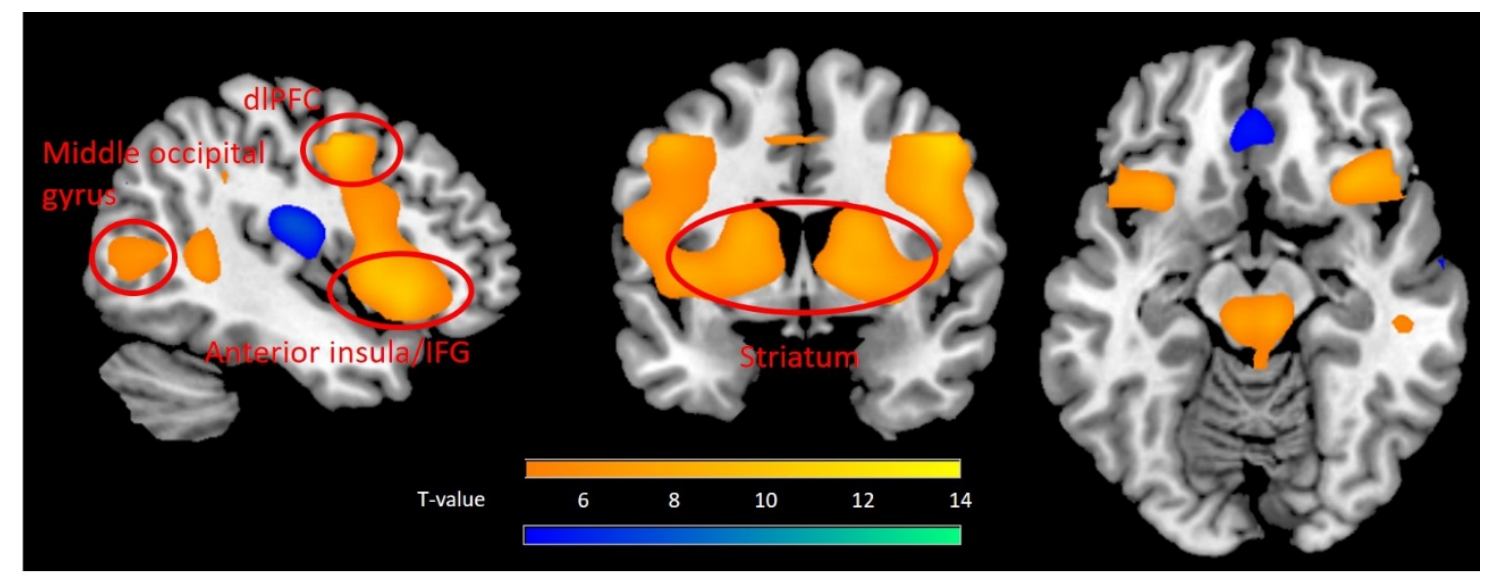

dIPFC [-42 -4 44]
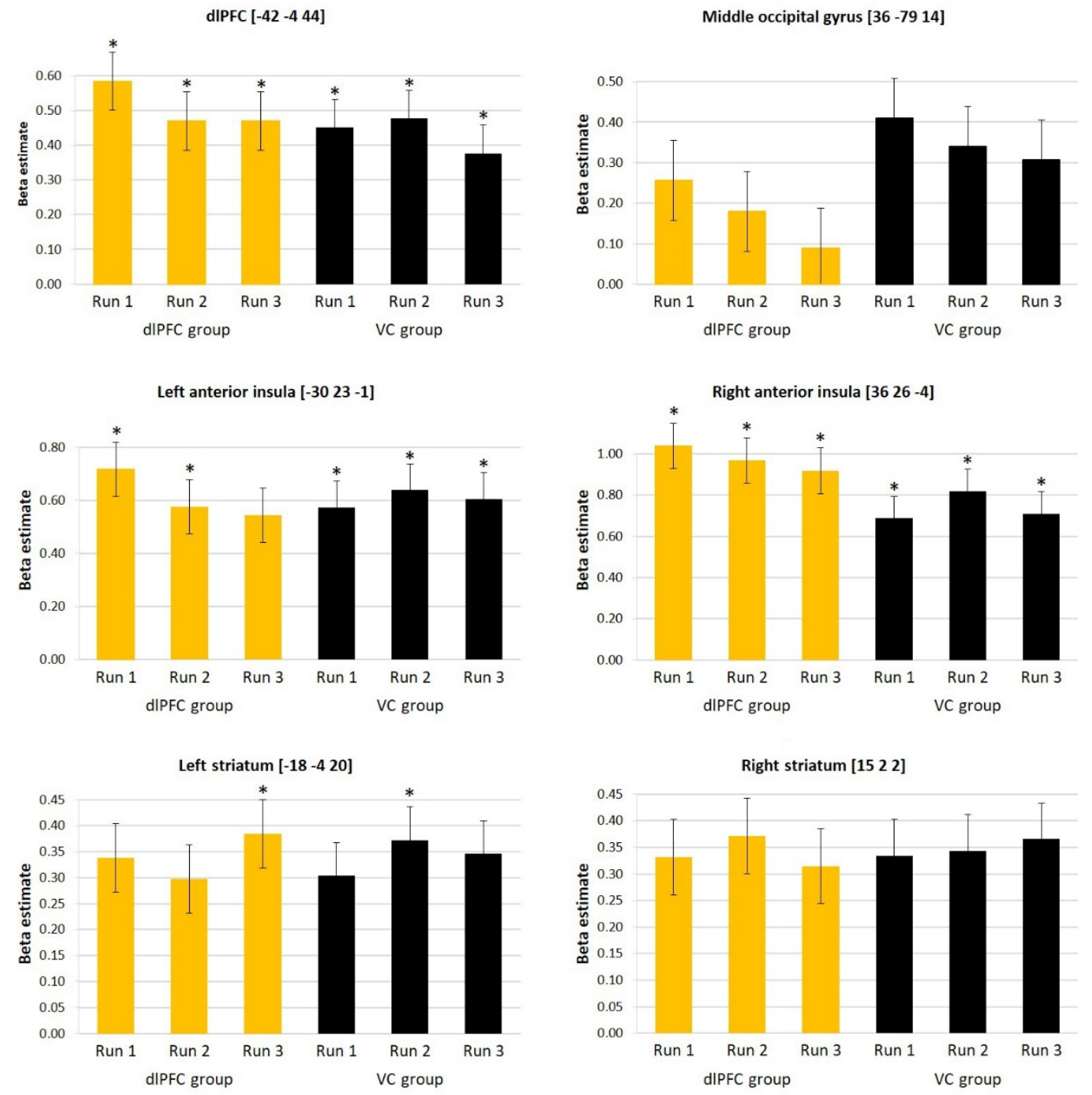

Figure 6. Main effect of rt-fMRI up-regulation training. Activation maps of up-regulation vs. passive viewing (orange-yellow) and the reversed contrast (blue-green) collapsed across all neurofeedback runs ( $p<$ 0.05 , FWE-corrected; $k>10$ ) plotted on a high-resolution anatomical T1-weighted template and displayed in neurological convention. Bottom panels depict average beta estimates and standard errors of the contrast up-regulation vs. passive viewing for all neurofeedback runs in dIPFC, visual cortex, insula and striatum. Coordinates in $\mathrm{MNI} ; * p<0.05 \mathrm{FWE}$-corrected and Bonferroni-corrected for number of tests (i.e., 36). 


\subsection{Functional connectivity during neurofeedback (offline analysis)}

Across groups, we found significantly increased connectivity between the left dlPFC and vmPFC during up-regulation compared to passing viewing $(\mathrm{t}(30)=3.60, p=0.003)$. Further significant positive connectivities were observed between left dlPFC and ACC $(\mathrm{t}(30)=4.42$, $p=0.0005)$ and between left dlPFC and ventral striatum $(\mathrm{t}(30)=2.57, p=0.024)$. A negative relationship was found between left dIPFC and inferior parietal cortex $(\mathrm{t}(30)=-5.03, p=$ 0.0003), superior parietal cortex $(\mathrm{t}(30)=-2.93, p=0.011)$, right BA46 $(\mathrm{t}(30)=-4.75, p=$ 0.0003), lateral occipital cortex $(\mathrm{t}(30)=-3.64, p=0.003)$, and right insula $(\mathrm{t}(30)=-3.23, p$ $=0.006$; see Figure 7). Comparisons between groups did not yield significant differences in connectivity. Although we found a trend towards stronger negative functional connectivity between dIPFC and right insula in the dIPFC compared to the VC group $(r=-0.33$ vs. $r=-$ $0.11, p=0.10$ ), these results indicate similar network patterns in both groups. 


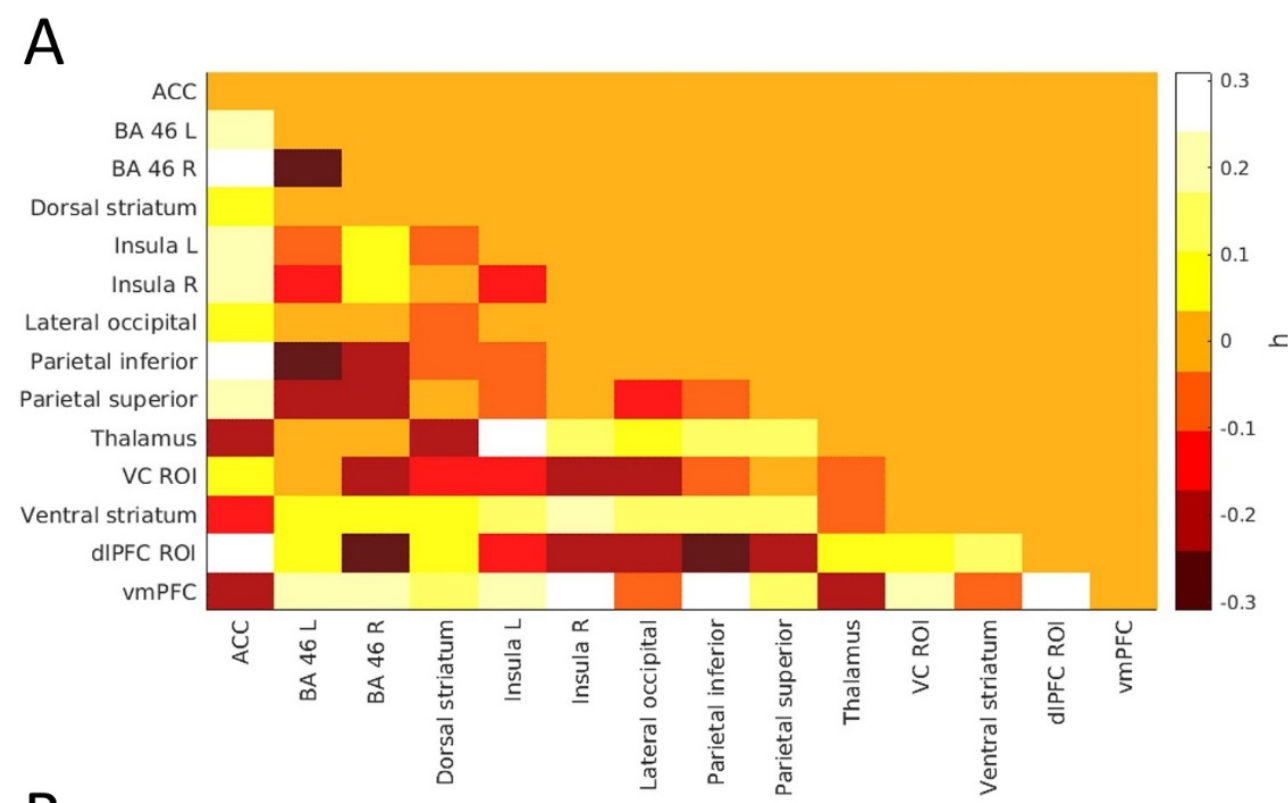

B

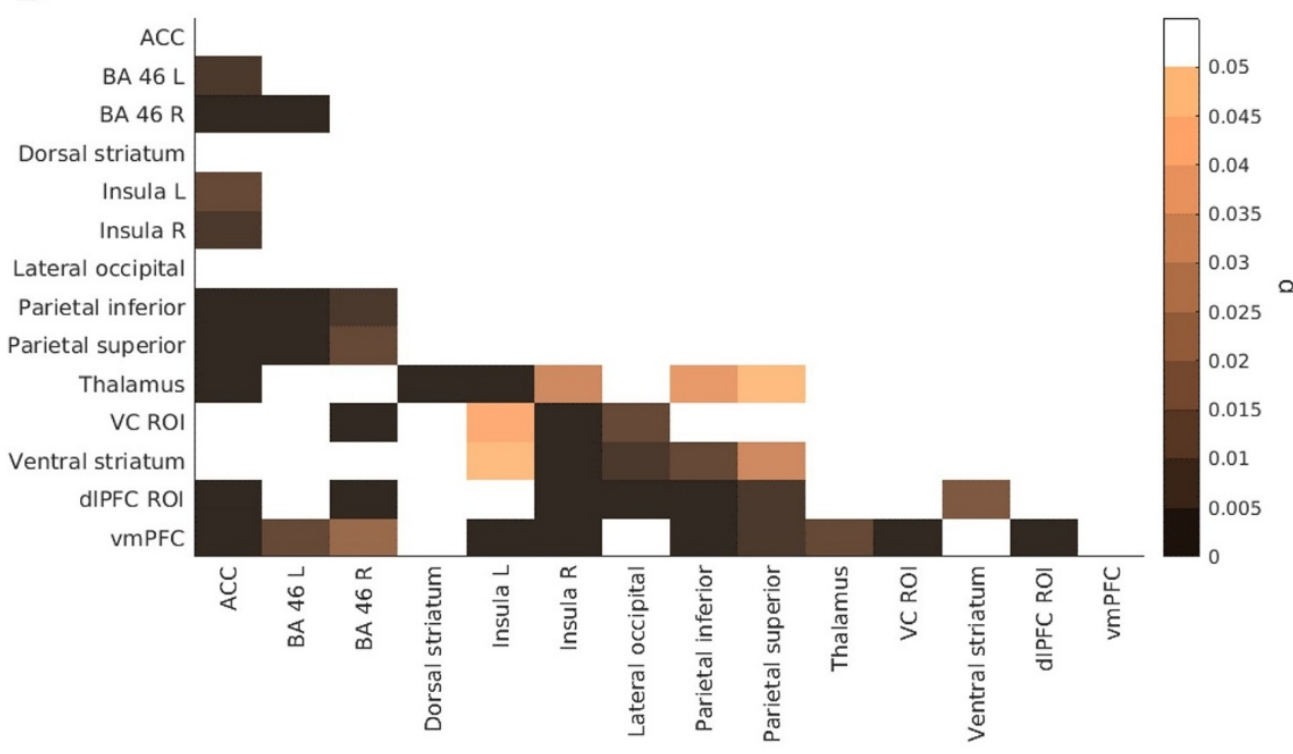

Figure 7. Task-based functional connectivity during up-regulation versus passive viewing independent of group. (A) h-values ( $h$ ) correspond to Fisher-z transformed pairwise correlations, (B) $p$ values (p) correspond to FDR-corrected $p$ values.

\subsection{Food selection task}

On a behavioral level, participants of both groups made less high-calorie selections directly after the neurofeedback training (dlPFC group: $29.71 \pm 4.22 \%$; VC group: $19.42 \pm 4.88 \%$ ) compared to before the training (dlPFC group: $42.78 \pm 4.72 \%$; VC group: $43.79 \pm 5.39 \%$ ), as confirmed by a significant effect of time $(F(1,30)=33.875, p<0.05)$. There was no significant group effect and only a trend-wise time $\times$ group interaction $(F(1,30)=3.324, p$ 
$=0.10$ ) suggesting that the decrease was slightly stronger in the VC group (Figure 8A). Due to technical problems data of three participants were missing for this task.

On the neural level, we did not find a group or group interaction effect. However, we identified stronger activation within the ventral anterior cingulate cortex extending to the dorsomedial PFC for the differential effect of high- vs. low-calorie food choices after neurofeedback training compared to before, as indicated by a significant time $\times$ choice interaction $(F(1,118)=16.03, p<0.05$, FWE-corrected, $\mathrm{k}=176)$ and a significant effect of high-calorie compared to low-calorie food choices in the post-training measurement, $(\mathrm{T}(1,118)=4.96, p<0.00001$, FWE-corrected, $\mathrm{k}=755)$. Moreover, this activation extended into the vmPFC (Figure 8B).

A

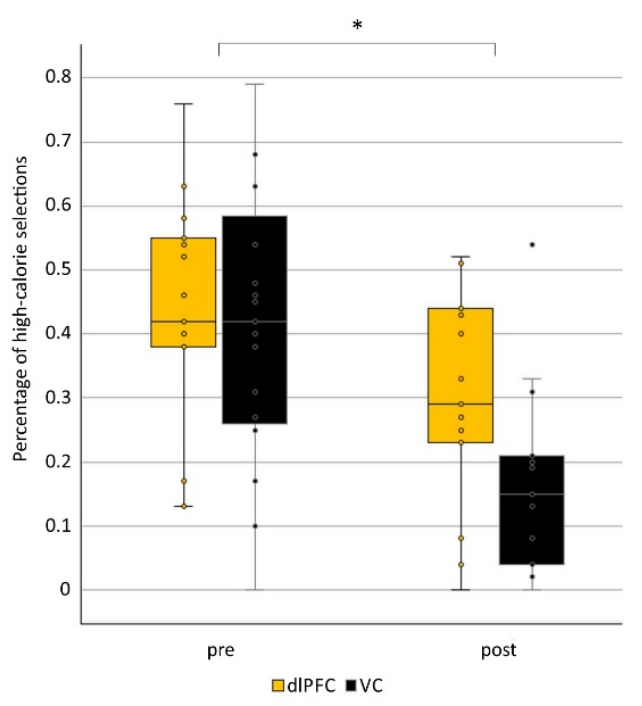

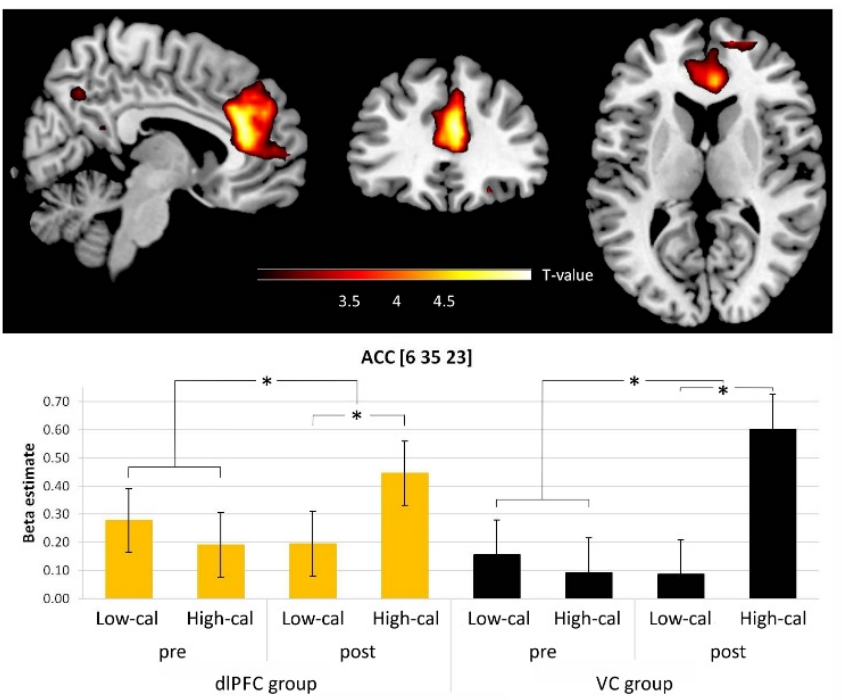

Figure 8. Results of the food selection task. (A) Percentage of high-calorie food choices before and after neurofeedback ( $\left.{ }^{*} p<0.05\right)$. (B) fMRI results showing the time $\times$ calorie interaction effect. For the purpose of presentation, activation of both groups is shown at an uncorrected $p<0.001$ and plotted on a highresolution anatomical T1-weighted template and displayed in neurological convention. Bottom panels show average beta estimates and standard errors for the anterior cingulate cortex (ACC); $p<0.05 \mathrm{FWE}-$ corrected.

\subsection{Food rating task}

Across both sessions participants of both groups rated high-calorie pictures as less healthy than low-calorie pictures $(F(1,33)=1479.106, p<0.001)$. No other main effects or interactions were significant with regard to healthiness ratings. 
Both groups rated high-calorie items as less palatable during the follow-up session (dlPFC group: $3.30 \pm 0.11$; VC-group: $3.16 \pm 0.11$ ) compared to the screening session (dlPFC group: $3.62 \pm 0.12$; VC group: $3.48 \pm 0.08$; Figure $9 \mathrm{~A}$ ), as also indicated by a significant main effect of time $F(1,33)=32.573, p<0.0001$, a significant main effect of calorie content, $F(1,33)$ $=40.056, p<0.0001$, and a significant time $\times$ calorie content interaction, $F(1,33)=21.637$, $p<.0001$. Simple main effects confirmed that the time $\times$ calorie content interaction was driven by a significant time effect for high-calorie food items $(F(1,33)=4.990, p<0.05)$. No group effects and no interactions with the factor group were observed.

With regard to food choices, both groups chose less high-calorie items during the follow-up session (dlPFC group: $42.50 \pm 4.96 \%$; VC-group: $33.89 \pm 4.54 \%$ ) compared to the screening session (dlPFC-group: $46.63 \pm 5.48 \%$; VC-group: $42.84 \pm 4.68 \%$; Figure 9B), as confirmed by a significant main effect of calorie content $(F(1,33)=58.557, p<0.001)$, a significant time $\times$ calorie content interaction $(F(1,33)=10.527, p<0.005)$, and simple main effects showing that the interaction was driven by a time effect for high-calorie food items $(F(1,33)=5.843, p<0.05)$. There were no significant time effects or group effects, no significant interactions between these factors, and no significant three-way interactions. As there were almost no yes-decisions for non-preferred low-calorie food items, only percentages of yes-decisions for preferred high-calorie food items were analyzed. Both groups made less yes-decisions for high-calorie food items that they preferred during the follow-up session (dlPFC-group: $61.19 \pm 6.50 \%$; VC-group: $51.26 \pm 7.24 \%$ ) compared to the screening session (dlPFC-group: $70.63 \pm 6.60 \%$; VC-group: $69.03 \pm 5.45 \% ; F(1,33)=$ 10.826, $p<0.005$ for time). No group effects or interaction effects were found.

In exploratory post-hoc analyses, we calculated correlation coefficients between training-induced changes in functional connectivity and effects on food ratings and food intake. We found that across groups, the changes in rated palatability of high-calorie food items in the follow-up session and in functional connectivity of dlPFC and insula activity during regulation were significantly correlated $(r=-0.506, p=0.003$; Bonferroni-corrected), indicating that the stronger the coupling between dIPFC and right insula, the stronger the decrease in rated palatability of high-calorie food items. 

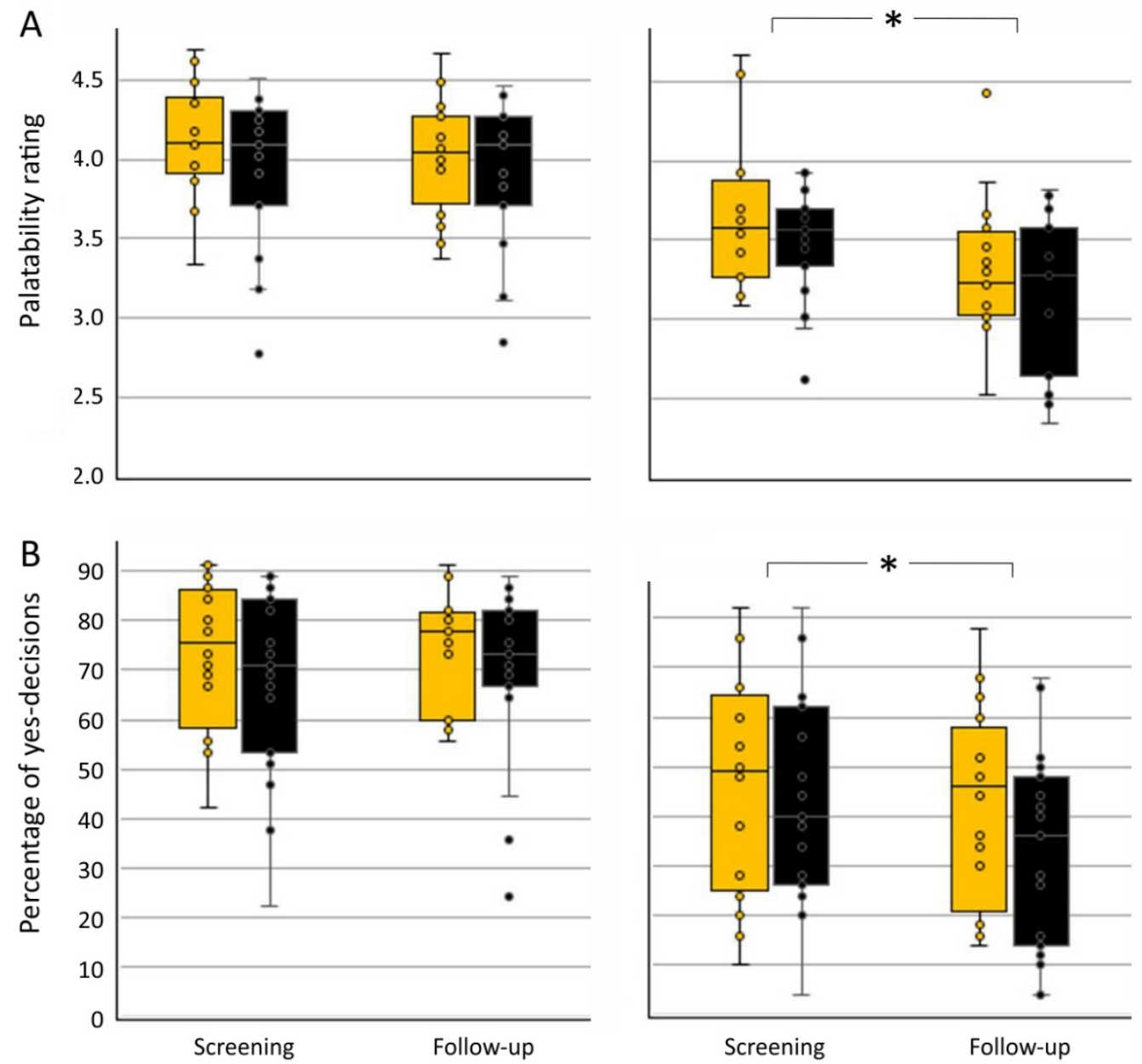

$\square$ dIPFC $\mathbf{a}$ VC

Figure 9. Results of the food rating task. Mean (A) palatability ratings and (B) percentages of yes-decisions for high and low-calorie food items during screening and the follow-up session; ${ }^{*} p<0.05$.

\subsection{Food intake}

As snack intake results were not normally distributed, we calculated a mixed ANOVA on ranks with the between-factor group and the within-factors time and taste. We found that across the three sessions, participants ate more chocolate cookies than neutral and salty snacks $(F(2,62)=99.464, p<0.0001$ for main effect of taste in contrast analyses). There were no other significant main effects or interactions with regard to snack intake.

Participants also rated the palatability and how often they would choose the respective snack if asked to. Directly after neurofeedback and at follow-up compared to screening, they rated the chocolate cookies as less palatable $(F(2,60)=5.455, p<0.01$ for taste and $F(4$, $120)=4.714, p<0.005$ for time $\times$ taste interaction). The same pattern was observed for the 
choice rating $(F(2,64)=6.752, p<0.01$ and $F(4,128)=5.749, p<0.005$, all GreenhouseGeisser-corrected). Simple main effects confirmed that the interaction effects were driven by the chocolate cookies $(F(2,62)=7.066, p<0.005$, and, respectively, $F(2,64)=6.864, p$ $<0.005$, for time). Moreover, participants would have chosen less of the chocolate and more of the rice snacks after neurofeedback compared to screening $(F(2,66)=3.468, p<0.05$, for simple main effect of rice snacks; see Table S5).

$$
\text { - Insert Supplementary Table S5 here - }
$$

\subsection{Body composition and eating-related questionnaires}

Table 1 summarizes body composition and questionnaire results of both groups at screening and the follow-up session. There were no significant differences between groups, and neither the VC nor the dlPFC group showed a significant change over time in any of these measures (all $p>0.05$ ). 
Table 1. Body composition and questionnaire results of both groups.

\begin{tabular}{|c|c|c|c|c|}
\hline & $\begin{array}{c}\text { dIPFC group } \\
(M \pm S E M)\end{array}$ & $\begin{array}{c}\text { VC group } \\
(M \pm S E M)\end{array}$ & $t$ & $p$ \\
\hline$N$ & 16 (4 male) & 19 (5 male) & & \\
\hline Age (years) & $29.25 \pm 1.93$ & $32.58 \pm 2.85$ & -0.93 & 0.360 \\
\hline Height (cm) & $170 \pm 2.34$ & $170.9 \pm 2.06$ & -0.29 & 0.775 \\
\hline Weight (kg) & $91.03 \pm 2.58$ & $91.5 \pm 2.44$ & -0.13 & 0.897 \\
\hline Weight (kg; follow-up) & $91.38 \pm 2.89$ & $91.37 \pm 2.47$ & 0.02 & 0.999 \\
\hline $\mathrm{BMI}\left(\mathrm{kg} / \mathrm{m}^{2}\right)$ & $31.63 \pm 0.91$ & $31.25 \pm 0.56$ & 0.37 & 0.715 \\
\hline BMI (kg/m²; follow-up) & $31.64 \pm 0.94$ & $31.32 \pm 0.53$ & 0.31 & 0.756 \\
\hline Body fat (\%) & $38.43 \pm 1.64$ & $37.15 \pm 1.40$ & 0.60 & 0.555 \\
\hline Body fat (\%; follow-up) & $38.76 \pm 1.54$ & $37.23 \pm 1.39$ & 0.74 & 0.466 \\
\hline FEV: Cognitive restraint & $8.63 \pm 1.02$ & $8.16 \pm 1.01$ & 0.32 & 0.748 \\
\hline FEV: Disinhibition & $7.44 \pm 0.72$ & $8.78 \pm 0.94$ & -1.11 & 0.278 \\
\hline FEV: Hunger & $7.06 \pm 0.82$ & $7.26 \pm 0.98$ & -0.15 & 0.879 \\
\hline FEV: Total & $23.13 \pm 1.86$ & $23.94 \pm 1.19$ & -0.28 & 0.748 \\
\hline PFS: Food available & $15.56 \pm 1.29$ & $14.26 \pm 1.19$ & 0.74 & 0.464 \\
\hline PFS: Food present & $13.31 \pm 0.68$ & $11.63 \pm 0.90$ & 1.45 & 0.158 \\
\hline PFS: Food tasted & $17.63 \pm 0.83$ & $15.68 \pm 0.83$ & 1.65 & 0.109 \\
\hline PFS: Total & $46.50 \pm 2.32$ & $41.58 \pm 2.68$ & 1.36 & 0.182 \\
\hline EDE: restraint & $1.36 \pm 0.29$ & $1.40 \pm 0.29$ & -0.91 & 0.928 \\
\hline EDE eating concern & $0.99 \pm 0.24$ & $1.05 \pm 0.32$ & -0.14 & 0.887 \\
\hline EDE weight concern & $3.03 \pm 0.38$ & $2.56 \pm 0.31$ & 0.96 & 0.344 \\
\hline EDE shape concern & $3.39 \pm 0.39$ & $2.96 \pm 0.33$ & 0.86 & 0.398 \\
\hline EDE average & $2.19 \pm 0.28$ & $1.99 \pm 0.25$ & 0.53 & 0.598 \\
\hline EDE restraint (follow-up) & $1.48 \pm 0.32$ & $1.44 \pm 0.32$ & 0.07 & 0.943 \\
\hline EDE eating concern (follow-up) & $1.06 \pm 0.30$ & $0.84 \pm 0.26$ & 0.56 & 0.579 \\
\hline EDE weigh concern (follow-up) & $2.90 \pm 0.36$ & $2.44 \pm 0.31$ & 0.97 & 0.340 \\
\hline EDE shape concern (follow-up) & $3.45 \pm 0.38$ & $3.01 \pm 0.34$ & 0.85 & 0.403 \\
\hline EDE average (follow-up) & $2.22 \pm 0.27$ & $1.94 \pm 0.27$ & 0.75 & 0.463 \\
\hline
\end{tabular}

BMI, body mass index; FEV, Fragebogen zum Essverhalten; PFS, Power of food scale; EDE, Eating Disorder Examination questionnaire; all values were obtained at screening except for those marked 'follow-up' (obtained during the follow-up session four weeks after the training). 
Hunger and satiety as assessed by visual analogue scales at the start of each session did not differ between groups (all $p>0.40$ ). Their results neither differed between sessions $(p>$ 0.09 ) or showed interactions between the factors group and time $(p>0.31)$. Analyses of the POMS questionnaire used to assess mood before and directly after neurofeedback training indicated a decrease in the scale depression/anxiety $(p<0.005)$ that appeared to be stronger in the dlPFC group (pre, $\mathrm{Mdn}=0.286$, post, $\mathrm{Mdn}=0.000 ; p<0.01$ ) than the VC group ( 0.143 to $0.237 ; p=0.096$, pre, $\mathrm{Mdn}=0.143$, post, $\mathrm{Mdn}=0.237$; post-hoc Wilcoxon signedrank tests). No significant pre-post changes and no differences between groups were found for the subscales vigor, fatigue or hostility. No training effects were observed for EDE scores (Table 1).

\subsection{Motivation, expectation and evaluation questionnaires}

We did not find indicators of group differences in terms of motivation to take part in the neurofeedback training inasmuch both groups reported to be highly motivated (dlPFC group, $4.46 \pm 0.20$; VC group, $4.63 \pm 0.13$ on a 5 -point scale). However, there was a significant difference regarding individual expectations towards the training, with the VC group reporting greater expectations than the dIPFC group (3.57 \pm 0.16 vs. $2.84 \pm 0.19, \mathrm{t}(27)=-$ $2.97 ; p<0.01$ ). The groups did not differ in their evaluation of the neurofeedback training at follow-up. They rated the neurofeedback training to be sufficiently efficient (dlPFC group, $2.55 \pm 0.16$; VC group, $2.50 \pm 0.18$ on a 5-point scale) and highly enjoyable (dlPFC group, $4.31 \pm 0.13$; VC group, $4.37 \pm 0.14$ )

Scores on the expectation scale correlated negatively with screening vs. follow-up differences in the choice of preferred high-calorie items $(r=-0.506, p=0.0051$; Bonferronicorrected). Separate analyses revealed that these correlations were more pronounced in the VC group $(r=-0.679, p=0.0054)$ while correlations were not significant, after Bonferronicorrection, for the dlPFC group.

Eight of the 16 participants of the dlPFC group and eight of the 19 participants of the VC group reported at follow-up to have used also in daily life the strategies applied during neurofeedback training. Five participants of the dlPFC group and one participant of the VC group reported to have used other strategies. 


\section{Discussion}

Our study demonstrates the feasibility and efficacy of rt-fMRI neurofeedback to improve food intake control in overweight and obese subjects; one session of neurofeedback training enabled participants to up-regulate dlPFC activity when confronted with palatable food items. The control group exhibited successful up-regulation of visual areas, the respective target region, but moreover also increased dlPFC activation. In both groups, we observed an increase in functional connectivity during up-regulation between dIPFC and areas involved in food value representation and reward processing. After training, both groups rated pictures of high-, but not low-calorie foods as less palatable and chose them less frequently compared to baseline. Actual snack intake remained unchanged, but chocolate cookies were rated as less palatable and less desirable. Self-reported expectations towards the neurofeedback training correlated with the decrease in preference for high-calorie foods in the control group, which might have contributed to the behavioral improvements seen in this group.

\subsection{Effects of neurofeedback training on brain activity}

During up-regulation training, participants showed activation of their target ROIs that, however, did not increase across training runs. We also found widespread activation changes during the regulation task in both groups, reflecting the effort to process feedback and reinforcement and apply and dynamically update mental strategies. A network of different brain areas was active during self-regulation, including anterior insula and dorsal striatum. The striatum is involved in skill as well as neurofeedback learning (Birbaumer et al., 2013; Sitaram et al., 2017) and respective activity increases may reflect learning aspects of successful neurofeedback-triggered self-regulation. The anterior insula is involved in a multitude of tasks including interoception and emotional awareness (Craig, 2009), and may also take part in reward processing during neurofeedback (Sitaram et al., 2017). Recent studies indicate that this structure primarily contributes to monitoring rather than selfregulating the feedback signal (Paret et al., 2018), which is in line with its role in the salience network and, consequently, for integration of information.

The dlPFC belongs to the left central executive network; it processes working memory contents (Bressler and Menon, 2010) and is considered a part of the neurofeedback control network (Sitaram et al., 2017). Indeed, a broad fronto-parietal and cingulo-opercular network of cognitive control is activated already during simple control tasks (Ninaus et al., 2013). Activation of the dIPFC in the VC and the dlPFC group alike may have been due to increased efforts to follow instructions and accomplish the task in the former in comparison 
to the latter group. It is also to note that alterations of visual networks such as increased global connectivity in the VC have been observed in obese individuals (Geha et al., 2017), along with increased resting state connectivity between dIPFC and VC (Moreno-Lopez et al., 2016), i.e., the training ROIs of our study. It is therefore conceivable that the modulation of visual areas affected the activity of other brain areas including the dIPFC, which would explain why the control group showed an effect of up-regulation not only in the VC, but also the dIPFC. Equivocal dlPFC activation in both groups may have triggered comparable behavioral changes, while the absence of incremental training effects across runs in both groups may have been due to particular efforts to up-regulate dIPFC activity in the beginning compared to later parts of the session. Although the dlPFC itself belongs to the neurofeedback control network, future studies might for example aim at down-regulation of the dIPFC as a control condition; however, this approach may frustrate participants and therefore raise ethical concerns (see Sorger et al., 2019; Thibault et al., 2017 for a discussion of this issue). Up to now, only few studies (Alegria et al., 2017; Mehler et al., 2018; Young et al., 2017) have used an approach similar to ours to assess the potential of neurofeedback in a randomized controlled clinical setting. That the control participants of these studies showed indication-specific behavioral improvements after receiving neurofeedback from an unrelated brain area is in line with our findings on neurofeedback-induced changes in food appraisal that are discussed below.

In analyses of network patterns, up-regulation compared to training was associated with increased connectivity between dIPFC and vmPFC in both groups. Given that this pattern reflects improved control of vmPFC-processed value signals by the dlPFC (Hare et al., 2009; Weygandt et al., 2013, 2015), our results indicate that neurofeedback training both of the dIPFC and of visual areas may target this neural substrate of self-control. In addition, we found significant increases in connectivity between dIPFC and ACC and, respectively, ventral striatum, i.e., parts of the neurofeedback reward processing network (Emmert et al., 2016; Sitaram et al., 2017). Interestingly, our results indicate that dlPFC-targeting neurofeedback training yields effects on connectivity and food choices similar to those of our previous approach that directly targeted functional connectivity (Spetter et al., 2017). This finding adds to the current discussion whether results of fMRI neurofeedback protocols based on functional connectivity, which are increasingly used (Kim et al., 2015; Koush et al., 2013; Megumi et al., 2015; Spetter et al., 2017; Yamashita et al., 2017), can likewise be achieved by single-ROI training approaches (e.g., Orlov et al., 2018; Papoutsi et al., 2018; Young et al., 2018). 


\subsection{Behavioral effects}

Immediately after neurofeedback (in the food selection task) as well as at follow-up (food rating task), participants reported reduced preference for high-calorie food items compared to baseline; they also rated chocolate cookies as less desirable. This pattern was reflected by a reduction in the number of "yes" decisions for high-calorie food items that received very high palatability ratings. Taken together, these results, which are in line with beneficial effects on eating behavior of transcranial direct current stimulation of dlPFC activity in obese subjects (Gluck et al., 2015; Heinitz et al., 2017; Montenegro et al., 2012), suggest improved self-control during food choices after the neurofeedback intervention in our obese and overweight individuals. While we did not observe significant correlations between selfregulation performance in individual training ROIs with pre-post differences in body weight, body fat, or behavioral outcomes, the strong correlation between the decrease in palatability ratings and functional connectivity-derived dlPFC-right insula regulation provides a direct link between training-induced neural changes and improvements in food intake control. Future neurofeedback studies may therefore specifically target this connectivity pattern. It might also be argued that behavioral outcomes emerged as an unspecific effect of the participants' effort to achieve the training goals. We found distinct differences in BOLD activation between high- and low-calorie food items in the ACC extending into the vmPFC after training, possibly reflecting the conflict between the two response alternatives (Botvinick et al., 2004). After neurofeedback training, selection of high-calorie food images therefore might have been associated with greater cognitive effort during the decision phase. However, higher activities in this region can be also related to the greater salience and reward expectance assigned to high-calorie food items (van der Laan et al., 2011) or their greater subjective stimulus value (Rangel, 2013), especially of items that the participants do not want to discard.

Surprisingly, these changes did not translate into reduced calorie intake in our covert assessment of snacking behavior when compared to baseline. Effects of anticipation might have masked potential outcomes of neurofeedback training. While subjects were not familiar with this task in the screening session, subjects had reason to anticipate the same test later on (based on the repetition of other behavioral tests across sessions). In conjunction with physiological anticipatory responses (Power and Schulkin, 2011), increased hunger might have counteracted any training effect in this regard. Alternatively, although the test was performed under the assumption of a taste assessment, subjects may have attenuated snack intake to comply with norms and social desirability, yielding relatively comparable intake 
across sessions and possibly also affecting subjective ratings of food palatability. Mere placebo effects of the neurofeedback interventions are less likely considering that in both groups, only around half of the subjects assumed to undergo efficient training.

Unspecific psychological factors play an important role in any kind of intervention or training. It has recently been shown for EEG neurofeedback that in blinded, placebocontrolled studies sham in comparison to genuine feedback can yield comparable behavioral effects and subjective changes (Schönenberg et al., 2017; Schabus et al, 2017). Therefore, we took care to detect the influence of expectation and motivation on behavioral outcomes. While both groups were highly motivated to participate, participants of the control group showed even greater expectations towards the neurofeedback training. Post-hoc correlations indeed suggest that the relatively enhanced expectance effects in the control group might have yielded behavioral improvements on par with the effects obtained in the dlPFC group. Of course, this explanation is tentative and underlines the need to control for unspecific psychological factors in rt-fMRI neurofeedback studies, particularly when considering potential clinical applications.

\subsection{Limitations and future directions}

The relatively limited duration of our intervention may have curbed its impact. However, while some clinical studies showed behavioral improvements only after three to four training sessions (Ruiz et al., 2011), others found strong training effects after a single day (Paret et al., 2014) or two days of training (Young et al., 2017). Also considering self-regulation performance, which in some cases even deteriorated after prolonged training (Hohenfeld et al., 2017), optimal training duration is in need of further investigation and, of course, may also depend on the particular target region or disorder. We did not include a separate transfer session without feedback to evaluate training success, instead relying on a food selection task to detect behavioral changes. For logistical reasons, the researchers in charge of neurofeedback training were not blinded regarding the subject's group assignment, but behavioral assessments were conducted by a separate, blinded investigator. As outlined above, our VC control group approach may have been too conservative considering that obese individuals show alterations in visual networks (Geha et al., 2017; Moreno-Lopez et al., 2016). Alternatively, the comparable results in the dIPFC and the VC group may indicate that it is the experience of successful neural regulation rather than changes in a specific target region that drives behavioral improvements (Alegria et al., 2017; Mehler et al., 2018). 
Alternative neurofeedback target regions to improve eating behavior in obesity include emotion- and reward-processing areas like the striatum, although many of these regions contribute to the neurofeedback learning process per se (Birbaumer et al., 2013; Emmert et al., 2017; Sitaram et al., 2017). Moreover, overeating in obesity may derive from hyper-responsivity or diminished sensitivity of reward-processing areas (Kenny, 2011), so that such approaches bear the risk of unintended effects. In this context, a non-controlled feasibility study in young healthy females by Ihssen and colleagues (2017) is worth mentioning that relied on 'motivational neurofeedback' from individually determined target areas, i.e., participants were instructed to downregulate brain activity elicited by high-calorie food cues that decreased in size if they succeeded. The authors found significant reductions in hunger ratings after neurofeedback but did not report any follow-up effects. Finally, less expensive forms of neurofeedback, such as EEG (Schmidt and Martin, 2016) or nearinfrared spectroscopy (NIRS; Barth et al., 2017; Hudak et al., 2017) might be promising approaches to improve self-control in obesity and accelerate the transfer into the clinical context. In sum, the results of this randomized controlled clinical study suggest that neurofeedback training to improve inhibitory self-control of eating behavior could be beneficial in multimodal strategies in the treatment of obesity.

\section{Acknowledgments}

This study was supported by funding within the framework of the F7 EU-project BRAINTRAIN (602186) and by grants from the German Federal Ministry of Education and Research (BMBF) to the German Center for Diabetes Research (DZD e.V.; 01GI0925).

We thank Verena Koppe for her most valuable contribution to data management. We also thank David Linden (School of Psychology, Cardiff University, UK) as well as Samantha Clarkstone, Kerenza Hood, Rachel McNamara, Rebecca Playle, Vincent Poile, Elizabeth Randell and Gareth Watson (Centre for Trials Research, College of Biomedical \& Life Sciences, Cardiff University, UK), and all BRAINTRAIN coordinators and collaborators for helpful and inspiring discussions.

\section{Disclosures}

Michael Lührs is an employee of the research company Brain Innovation B.V., Maastricht, The Netherlands. All other authors have nothing to disclose. 


\section{References}

Alegria, A.A., Wulff, M., Brinson, H., Barker, G.J., Norman, L.J., Brandeis, D., Stahl, D., David, A.S., Taylor, E., Giampietro, V., Rubia, K., 2017. Real-time fMRI neurofeedback in adolescents with attention deficit hyperactivity disorder. Hum. Brain Mapp. 38, 3190-3209. https://doi.org/10.1002/hbm.23584

Barth, B., Hudak, J., Blume, F., Marx, A.M., Mayer, K., Strehl, U., Ehlis, A.C., Fallgatter, A., 2017. NIRS neurofeedback in patients with ADHD. Eur. Neuropsychopharmacol. 27, S563. https://doi.org/10.1016/S0924-977X(17)31083-0

Bartholdy, S., Dalton, B., O’Daly, O.G., Campbell, I.C., Schmidt, U., 2016. A systematic review of the relationship between eating, weight and inhibitory control using the stop signal task. Neurosci. Biobehav. Rev. 64, 35-62. https://doi.org/10.1016/j.neubiorev.2016.02.010

Bassett, D.S., Khambhati, A.N., 2017. A network engineering perspective on probing and perturbing cognition with neurofeedback. Ann. N. Y. Acad. Sci. 1396, 126-143. https://doi.org/10.1111/nyas.13338

Batterink, L., Yokum, S., Stice, E., 2010. Body mass correlates inversely with inhibitory control in response to food among adolescent girls: An fMRI study. Neuroimage 52, 1696-1703. https://doi.org/10.1016/j.neuroimage.2010.05.059

Berthoud, H.-R., Zheng, H., 2012. Modulation of taste responsiveness and food preference by obesity and weight loss. Physiol. Behav. 107, 527-532. https://doi.org/10.1016/j.physbeh.2012.04.004

Birbaumer, N., Ruiz, S., Sitaram, R., 2013. Learned regulation of brain metabolism. Trends Cogn. Sci. 17, 295302. https://doi.org/10.1016/j.tics.2013.04.009

Botvinick, M.M., Cohen, J.D., Carter, C.S., 2004. Conflict monitoring and anterior cingulate cortex: an update. Trends Cogn. Sci. 8, 539-546. https://doi.org/10.1016/j.tics.2004.10.003

Bray, G.A., 2004. Medical consequences of obesity. J. Clin. Endocrinol. Metab. 89, 2583-2589. https://doi.org/10.1210/jc.2004-0535

Bressler, S.L., Menon, V., 2010. Large-scale brain networks in cognition: emerging methods and principles. Trends Cogn. Sci. 14, 277-290. https://doi.org/10.1016/j.tics.2010.04.004

Brooks, S.J., Cedernaes, J., Schiöth, H.B., 2013. Increased Prefrontal and Parahippocampal Activation with Reduced Dorsolateral Prefrontal and Insular Cortex Activation to Food Images in Obesity: A MetaAnalysis of fMRI Studies. PLoS One 8, e60393. https://doi.org/10.1371/journal.pone.0060393

Charbonnier, L., van Meer, F., van der Laan, L.N., Viergever, M.A., Smeets, P.A.M., 2015. Standardized food images: A photographing protocol and image database. Appetite 96, 166-173. https://doi.org/10.1016/j.appet.2015.08.041

Collaborators, T.G. 2015 O., 2017. Health Effects of Overweight and Obesity in 195 Countries over 25 Years. N. Engl. J. Med. 377, 13-27. https://doi.org/10.1056/NEJMoa1614362

Craig, A.D., 2009. How do you feel - now? The anterior insula and human awareness. Nat. Rev. Neurosci. https://doi.org/10.1038/nrn2555

DelParigi, a, Chen, K., Salbe, a D., Hill, J.O., Wing, R.R., Reiman, E.M., Tataranni, P. a, 2007. Successful dieters have increased neural activity in cortical areas involved in the control of behavior. Int. J. Obes. (Lond). 31, 440-8. https://doi.org/10.1038/sj.ijo.0803431

Emmert, K., Kopel, R., Sulzer, J., Brühl, A.B., Berman, B.D., Linden, D.E.J., Horovitz, S.G., Breimhorst, M., Caria, A., Frank, S., Johnston, S., Long, Z., Paret, C., Robineau, F., Veit, R., Bartsch, A., Beckmann, C.F., Van De Ville, D., Haller, S., 2016. Meta-analysis of real-time fMRI neurofeedback studies using individual participant data: How is brain regulation mediated? Neuroimage 124, 806-812. https://doi.org/10.1016/j.neuroimage.2015.09.042

Flegal, D.K.M., Kit, D.B.K., Orpana, D.H., Graubard, D.B.I., 2013. Association of All-Cause Mortality With Overweight and Obesity Using Standard Body Mass Index Categories: A Systematic Review and Metaanalysis. JAMA 309, 71. https://doi.org/10.1001/JAMA.2012.113905

Frank, S., Lee, S., Preissl, H., Schultes, B., Birbaumer, N., Veit, R., 2012. The obese brain athlete: Selfregulation of the anterior insula in adiposity. PLoS One 7, e42570. https://doi.org/10.1371/journal.pone.0042570

Geha, P., Cecchi, G., Todd Constable, R., Abdallah, C., Small, D.M., 2017. Reorganization of brain connectivity in obesity. Hum. Brain Mapp. 38, 1403-1420. https://doi.org/10.1002/hbm.23462

Gluck, M.E., Alonso-Alonso, M., Piaggi, P., Weise, C.M., Jumpertz-Von Schwartzenberg, R., Reinhardt, M., Wassermann, E.M., Venti, C.A., Votruba, S.B., Krakoff, J., 2015. Neuromodulation targeted to the prefrontal cortex induces changes in energy intake and weight loss in obesity. Obesity 23, 2149-2156. https://doi.org/10.1002/oby.21313

Goldman, R.L., Borckardt, J.J., Frohman, H.A., O’Neil, P.M., Madan, A., Campbell, L.K., Budak, A., George, M.S., 2011. Prefrontal cortex transcranial direct current stimulation (tDCS) temporarily reduces food cravings and increases the self-reported ability to resist food in adults with frequent food craving. Appetite 56, 741-746. https://doi.org/10.1016/j.appet.2011.02.013 
Greer, S.M., Trujillo, A.J., Glover, G.H., Knutson, B., 2014. Control of nucleus accumbens activity with neurofeedback. Neuroimage 96, 237-244. https://doi.org/10.1016/j.neuroimage.2014.03.073

Hallschmid, M., Higgs, S., Thienel, M., Ott, V., Lehnert, H., 2012. Postprandial administration of intranasal insulin intensifies satiety and reduces intake of palatable snacks in women. Diabetes 61, 782-789. https://doi.org/10.2337/db11-1390

Hare, T.A., Camerer, C.F., Rangel, A., 2009. Self-control in decision-making involves modulation of the vmPFC valuation system. Science 324, 646-8. https://doi.org/10.1126/science.1168450

Harmelech, T., Friedman, D., Malach, R., 2015. Differential magnetic resonance neurofeedback modulations across extrinsic (visual) and intrinsic (default-mode) nodes of the human cortex. J. Neurosci. 35, $2588-95$. https://doi.org/10.1523/JNEUROSCI.3098-14.2015

Hege, M.A., Stingl, K.T., Kullmann, S., Schag, K., Giel, K.E., Zipfel, S., Preissl, H., 2015. Attentional impulsivity in binge eating disorder modulates response inhibition performance and frontal brain networks. Int. J. Obes. 39, 353-360. https://doi.org/10.1038/ijo.2014.99

Heinitz, S., Reinhardt, M., Piaggi, P., Weise, C.M., Diaz, E., Stinson, E.J., Venti, C., Votruba, S.B., Wassermann, E.M., Alonso-Alonso, M., Krakoff, J., Gluck, M.E., 2017. Neuromodulation directed at the prefrontal cortex of subjects with obesity reduces snack food intake and hunger in a randomized trial. Am. J. Clin. Nutr. 106, 1347-1357. https://doi.org/10.3945/ajcn.117.158089

Higgs, S., Williamson, A.C., Attwood, A.S., 2008. Recall of recent lunch and its effect on subsequent snack intake. Physiol. Behav. 94, 454-462. https://doi.org/10.1016/j.physbeh.2008.02.011

Hilbert, A., Tuschen-Caffier, B., 2011. Eating Disorder Examination - Questionnaire. Münster Verlag für Psychother. 1-30. https://doi.org/10.1037/t03975-000

Hohenfeld, C., Nellessen, N., Dogan, I., Kuhn, H., Müller, C., Papa, F., Ketteler, S., Goebel, R., Heinecke, A., Shah, N.J., Schulz, J.B., Reske, M., Reetz, K., 2017. Cognitive improvement and brain changes after realtime functional MRI neurofeedback training in healthy elderly and prodromal Alzheimer's disease. Front. Neurol. 8, 384. https://doi.org/10.3389/fneur.2017.00384

Hollmann, M., Hellrung, L., Pleger, B., Schlögl, H., Kabisch, S., Stumvoll, M., Villringer, A., Horstmann, A., 2012. Neural correlates of the volitional regulation of the desire for food. Int. J. Obes. 36, 648-655. https://doi.org/10.1038/ijo.2011.125

Ihssen, N., Sokunbi, M.O., Lawrence, A.D., Lawrence, N.S., Linden, D.E.J., 2017. Neurofeedback of visual food cue reactivity: a potential avenue to alter incentive sensitization and craving. Brain Imaging Behav. 11, 915-924. https://doi.org/10.1007/s11682-016-9558-x

Jensen, C.D., Kirwan, C.B., 2015. Functional brain response to food images in successful adolescent weight losers compared with normal-weight and overweight controls. Obesity 23, 630-636. https://doi.org/10.1002/oby.21004

Jensen, M.D., Ryan, D.H., Apovian, C.M., Ard, J.D., Comuzzie, A.G., Donato, K.A., Hu, F.B., Hubbard, V.S., Jakicic, J.M., Kushner, R.F., Loria, C.M., Millen, B.E., Nonas, C.A., Pi-Sunyer, F.X., Stevens, J., Stevens, V.J., Wadden, T.A., Wolfe, B.M., Yanovski, S.Z., Jordan, H.S., Kendall, K.A., Lux, L.J., Mentor-Marcel, R., Morgan, L.C., Trisolini, M.G., Wnek, J., Anderson, J.L., Halperin, J.L., Albert, N.M., Bozkurt, B., Brindis, R.G., Curtis, L.H., DeMets, D., Hochman, J.S., Kovacs, R.J., Ohman, E.M., Pressler, S.J., Sellke, F.W., Shen, W.-K., Smith, S.C., Tomaselli, G.F., American College of Cardiology/American Heart Association Task Force on Practice Guidelines, W.-K., Obesity Society, S.O.P., Smith, S.C., Jr, Tomaselli, G.F., 2014. 2013 AHA/ACC/TOS guideline for the management of overweight and obesity in adults: a report of the American College of Cardiology/American Heart Association Task Force on Practice Guidelines and The Obesity Society. Circulation 129, S102-38. https://doi.org/10.1161/01.cir.0000437739.71477.ee

Johnson, K.A., Hartwell, K., Lematty, T., Borckardt, J., Morgan, P.S., Govindarajan, K., Brady, K., George, M.S., 2012. Intermittent "Real-time” fMRI Feedback Is Superior to Continuous Presentation for a Motor Imagery Task: A Pilot Study. J. Neuroimaging 22, 58-66. https://doi.org/10.1111/j.15526569.2010.00529.x

Kenny, P. J., 2011. Reward mechanisms in obesity: new insights and future directions. Neuron, 69(4), 664-679. https://doi.org/10.1016/j.neuron.2011.02.016

Kim, D.-Y., Yoo, S.-S., Tegethoff, M., Meinlschmidt, G., Lee, J.-H., 2015. The Inclusion of Functional Connectivity Information into fMRI-based Neurofeedback Improves Its Efficacy in the Reduction of Cigarette Cravings. J. Cogn. Neurosci. 27, 1552-1572. https://doi.org/10.1162/jocn_a_00802

Koush, Y., Rosa, M.J., Robineau, F., Heinen, K., W Rieger, S., Weiskopf, N., Vuilleumier, P., Van De Ville, D., Scharnowski, F., 2013. Connectivity-based neurofeedback: dynamic causal modeling for real-time fMRI. Neuroimage 81, 422-30. https://doi.org/10.1016/j.neuroimage.2013.05.010

Lavagnino, L., Arnone, D., Cao, B., Soares, J.C., Selvaraj, S., 2016. Inhibitory control in obesity and binge eating disorder: A systematic review and meta-analysis of neurocognitive and neuroimaging studies. Neurosci. Biobehav. Rev. 68, 714-726. https://doi.org/10.1016/j.neubiorev.2016.06.041

Le, D.S.N.T., Pannacciulli, N., Chen, K., Del Parigi, A., Salbe, A.D., Reiman, E.M., Krakoff, J., 2006. Less activation of the left dorsolateral prefrontal cortex in response to a meal: A feature of obesity. Am. J. Clin. 
Nutr. 84, 725-731. https://doi.org/84/4/725 [pii]

Le, D.S.N.T., Pannacciulli, N., Chen, K., Salbe, A.D., Hill, J.O., Wing, R.R., Reiman, E.M., Krakoff, J., 2007.

Less activation in the left dorsolateral prefrontal cortex in the reanalysis of the response to a meal in obese than in lean women and its association with successful weight loss. Am. J. Clin. Nutr. 86, 573-579. https://doi.org/86/3/573 [pii]

Lehnert, T., Sonntag, D., Konnopka, A., Riedel-Heller, S., König, H.-H., 2013. Economic costs of overweight and obesity. Best Pract. Res. Clin. Endocrinol. Metab. 27, 105-115. https://doi.org/10.1016/j.beem.2013.01.002

Lowe, M.R., Butryn, M.L., 2007. Hedonic hunger: A new dimension of appetite? Physiol. Behav. 91, 432-439. https://doi.org/10.1016/J.PHYSBEH.2007.04.006

McCaffery, J.M., Haley, A.P., Sweet, L.H., Phelan, S., Raynor, H.A., Del Parigi, A., Cohen, R., Wing, R.R., 2009. Differential functional magnetic resonance imaging response to food pictures in successful weightloss maintainers relative to normal-weight and obese controls. Am. J. Clin. Nutr. 90, 928-934. https://doi.org/10.3945/ajcn.2009.27924

McNair, D., Lorr, M., Droppleman, L., 1981. POMS Profile of Mood States. Ein Verfahren zur Messung von Stimmungszuständen. Int. Skalen für Psychiatr.

Megumi, F., Yamashita, A., Kawato, M., Imamizu, H., 2015. Functional MRI neurofeedback training on connectivity between two regions induces long-lasting changes in intrinsic functional network. Front. Hum. Neurosci. 9, 160. https://doi.org/10.3389/fnhum.2015.00160

Mehler, D.M.A., Sokunbi, M.O., Habes, I., Barawi, K., Subramanian, L., Range, M., Evans, J., Hood, K., Lührs, M., Keedwell, P., Goebel, R., Linden, D.E.J., 2018. Targeting the affective brain—a randomized controlled trial of real-time fMRI neurofeedback in patients with depression. Neuropsychopharmacology 1-8. https://doi.org/10.1038/s41386-018-0126-5

Montenegro, R.A., Okano, A.H., Cunha, F.A., Gurgel, J.L., Fontes, E.B., Farinatti, P.T. V, 2012. Prefrontal cortex transcranial direct current stimulation associated with aerobic exercise change aspects of appetite sensation in overweight adults. Appetite 58, 333-338. https://doi.org/10.1016/j.appet.2011.11.008

Moreno-Lopez, L., Contreras-Rodriguez, O., Soriano-Mas, C., Stamatakis, E.A., Verdejo-Garcia, A., 2016. Disrupted functional connectivity in adolescent obesity. NeuroImage Clin. 12, 262-268. https://doi.org/10.1016/j.nicl.2016.07.005

Nijs, I.M.T., Franken, I.H.A., Muris, P., 2010. Food-related Stroop interference in obese and normal-weight individuals: Behavioral and electrophysiological indices. Eat. Behav. 11, 258-265. https://doi.org/10.1016/j.eatbeh.2010.07.002

Ninaus, M., Kober, S.E., Witte, M., Koschutnig, K., Stangl, M., Neuper, C., Wood, G., 2013. Neural substrates of cognitive control under the belief of getting neurofeedback training. Front. Hum. Neurosci. 7, 1-10. https://doi.org/10.3389/fnhum.2013.00914

Orlov, N.D., Giampietro, V., O’Daly, O., Lam, S.-L., Barker, G.J., Rubia, K., McGuire, P., Shergill, S.S., Allen, P., 2018. Real-time fMRI neurofeedback to down-regulate superior temporal gyrus activity in patients with schizophrenia and auditory hallucinations: a proof-of-concept study. Transl. Psychiatry 8, 46. https://doi.org/10.1038/s41398-017-0067-5

Ott, V., Finlayson, G., Lehnert, H., Heitmann, B., Heinrichs, M., Born, J., Hallschmid, M., 2013. Oxytocin reduces reward-driven food intake in humans. Diabetes 62, 3418-3425. https://doi.org/10.2337/db13-0663

Papoutsi, M., Weiskopf, N., Langbehn, D., Reilmann, R., Rees, G., Tabrizi, S.J., 2018. Stimulating neural plasticity with real-time fMRI neurofeedback in Huntington's disease: A proof of concept study. Hum. Brain Mapp. 39, 1339-1353. https://doi.org/10.1002/hbm.23921

Paret, C., Kluetsch, R., Ruf, M., Demirakca, T., Hoesterey, S., Ende, G., Schmahl, C., 2014. Down-regulation of amygdala activation with real-time fMRI neurofeedback in a healthy female sample. Front. Behav. Neurosci. 8, 299. https://doi.org/10.3389/fnbeh.2014.00299

Paret, C., Zähringer, J., Ruf, M., Gerchen, M.F., Mall, S., Hendler, T., Schmahl, C., Ende, G., 2018. Monitoring and control of amygdala neurofeedback involves distributed information processing in the human brain. Hum. Brain Mapp. https://doi.org/10.1002/hbm.24057

Power, M.L., Schulkin, J., 2011. Anticipatory Physiological Regulation in Feeding Biology. Appetite 50, 829844. https://doi.org/10.1007/978-0-387-92271-3

Pudel, V., Westenhoefer, J., 1989. Fragebogen zum Essverhalten. Göttingen: Hogrefe.

Rangel, A., 2013. Regulation of dietary choice by the decision-making circuitry. Nat. Neurosci. https://doi.org/10.1038/nn.3561

Ruiz, S., Lee, S., Soekadar, S.R., Caria, A., Veit, R., Kircher, T., Birbaumer, N., Sitaram, R., 2011. Acquired self-control of insula cortex modulates emotion recognition and brain network connectivity in schizophrenia. Hum. Brain Mapp. 34, 200-212. https://doi.org/10.1002/hbm.21427

Scharnowski, F., Hutton, C., Josephs, O., Weiskopf, N., Rees, G., 2012. Improving Visual Perception through Neurofeedback. J. Neurosci. 32, 17830-17841. https://doi.org/10.1523/JNEUROSCI.6334-11.2012

Schmidt, J., Martin, A., 2016. Neurofeedback Against Binge Eating: A Randomized Controlled Trial in a Female Subclinical Threshold Sample. Eur. Eat. Disord. Rev. 24, 406-416. https://doi.org/10.1002/erv.2453 
Schönenberg, M., Wiedemann, E., Schneidt, A., Scheeff, J., Logemann, A., Keune, P.M., Hautzinger, M., 2017. Neurofeedback, sham neurofeedback, and cognitive-behavioural group therapy in adults with attentiondeficit hyperactivity disorder: a triple-blind, randomised, controlled trial. The lancet. Psychiatry 4, 673684. https://doi.org/10.1016/S2215-0366(17)30291-2

Sherlin, L.H., Arns, M., Lubar, J., Heinrich, H., Kerson, C., Strehl, U., Sterman, M.B., 2011. Neurofeedback and Basic Learning Theory: Implications for Research and Practice. J. Neurother. 15, 292-304. https://doi.org/10.1080/10874208.2011.623089

Shibata, K., Watanabe, T., Sasaki, Y., Kawato, M., 2011. Perceptual Learning Incepted by Decoded fMRI Neurofeedback Without Stimulus Presentation. Science (80-. ). 334, 1413-1415. https://doi.org/10.1126/science.1212003

Sitaram, R., Ros, T., Stoeckel, L., Haller, S., Scharnowski, F., Lewis-Peacock, J., Weiskopf, N., Blefari, M.L., Rana, M., Oblak, E., Birbaumer, N., Sulzer, J., 2017. Closed-loop brain training: the science of neurofeedback. Nat. Rev. Neurosci. 18, 86-100. https://doi.org/10.1038/nrn.2016.164

Sorger, B., Scharnowski, F., Linden, D.E.J., Hampson, M., Young, K.D., 2019. Control freaks: Towards optimal selection of control conditions for fMRI neurofeedback studies. Neuroimage 186, 256-265. https://doi.org/10.1016/J.NEUROIMAGE.2018.11.004

Spetter, M.S., Malekshahi, R., Birbaumer, N., Lührs, M., van der Veer, A.H., Scheffler, K., Spuckti, S., Preissl, H., Veit, R., Hallschmid, M., 2017. Volitional regulation of brain responses to food stimuli in overweight and obese subjects: A real-time fMRI feedback study. Appetite 112, 188-195. https://doi.org/10.1016/j.appet.2017.01.032

Strehl, U., 2014. What learning theories can teach us in designing neurofeedback treatments. Front. Hum. Neurosci. 8, 894. https://doi.org/10.3389/fnhum.2014.00894

Thibault, R.T., MacPherson, A., Lifshitz, M., Roth, R.R., Raz, A., 2017. Neurofeedback with fMRI: A critical systematic review. Neuroimage. https://doi.org/10.1016/j.neuroimage.2017.12.071

Tzourio-Mazoyer, N., Landeau, B., Papathanassiou, D., Crivello, F., Etard, O., Delcroix, N., Mazoyer, B., Joliot, M., 2002. Automated Anatomical Labeling of Activations in SPM Using a Macroscopic Anatomical Parcellation of the MNI MRI Single-Subject Brain. Neuroimage 15, 273-289. https://doi.org/10.1006/NIMG.2001.0978

van der Laan, L.N., de Ridder, D.T.D., Viergever, M.A., Smeets, P.A.M., 2011. The first taste is always with the eyes: A meta-analysis on the neural correlates of processing visual food cues. Neuroimage 55, 296-303. https://doi.org/10.1016/j.neuroimage.2010.11.055

Volkow, N.D., Wang, G.-J., Telang, F., Fowler, J.S., Goldstein, R.Z., Alia-Klein, N., Logan, J., Wong, C., Thanos, P.K., Ma, Y., Pradhan, K., 2009. Inverse Association Between BMI and Prefrontal Metabolic Activity in Healthy Adults. Obesity 17, 60-65. https://doi.org/10.1038/oby.2008.469

Weiskopf, N., 2012. Real-time fMRI and its application to neurofeedback. Neuroimage 62, 682-692. https://doi.org/10.1016/j.neuroimage.2011.10.009

Weiskopf, N., Sitaram, R., Josephs, O., Veit, R., Scharnowski, F., Goebel, R., Birbaumer, N., Deichmann, R., Mathiak, K., 2007. Real-time functional magnetic resonance imaging: methods and applications. Magn. Reson. Imaging 25, 989-1003. https://doi.org/10.1016/j.mri.2007.02.007

Weygandt, M., Mai, K., Dommes, E., Leupelt, V., Hackmack, K., Kahnt, T., Rothemund, Y., Spranger, J., Haynes, J.D., 2013. The role of neural impulse control mechanisms for dietary success in obesity. Neuroimage 83, 669-678. https://doi.org/10.1016/j.neuroimage.2013.07.028

Weygandt, M., Mai, K., Dommes, E., Ritter, K., Leupelt, V., Spranger, J., Haynes, J.D., 2015. Impulse control in the dorsolateral prefrontal cortex counteracts post-diet weight regain in obesity. Neuroimage 109, 318327. https://doi.org/10.1016/j.neuroimage.2014.12.073

Whitfield-Gabrieli, S., Nieto-Castanon, A., 2012. Conn : A Functional Connectivity Toolbox for Correlated and Anticorrelated Brain Networks. Brain Connect. 2, 125-141. https://doi.org/10.1089/brain.2012.0073

Yamashita, A., Hayasaka, S., Kawato, M., Imamizu, H., 2017. Connectivity Neurofeedback Training Can Differentially Change Functional Connectivity and Cognitive Performance. Cereb. Cortex 27, 4960-4970. https://doi.org/10.1093/cercor/bhx177

Yokum, S., Stice, E., 2013. Cognitive regulation of food craving: Effects of three cognitive reappraisal strategies on neural response to palatable foods. Int. J. Obes. 37, 1565-1570. https://doi.org/10.1038/ijo.2013.39

Young, K.D., Siegle, G.J., Misaki, M., Zotev, V., Phillips, R., Drevets, W.C., Bodurka, J., 2018. Altered taskbased and resting-state amygdala functional connectivity following real-time fMRI amygdala neurofeedback training in major depressive disorder. NeuroImage. Clin. 17, 691-703. https://doi.org/10.1016/j.nicl.2017.12.004

Young, K.D., Siegle, G.J., Zotev, V., Phillips, R., Misaki, M., Yuan, H., Drevets, W.C., Bodurka, J., 2017. Randomized Clinical Trial of Real-Time fMRI Amygdala Neurofeedback for Major Depressive Disorder: Effects on Symptoms and Autobiographical Memory Recall. Am. J. Psychiatry 174, 748-755. https://doi.org/10.1176/appi.ajp.2017.16060637 